\title{
The Development of Auger Spectroscopy as a Probe of Local Electronic Structure
}

\author{
Peter Weightman
}

Physics Department and-IRC in Surface Science, University of Liverpool, Oxford St. Liverpool L69 3BX, UK

(Received May 2; accepted May 17, 1995)

\begin{abstract}
This review is concerned with the development of Auger spectroscopy as a probe of local electronic structure. The contribution that CCC, CCV and CVV Auger transitions can make to the study of electronic structure is reviewed and the appropriate theoretical context for the interpretation of the spectra produced by each process is discussed. It is shown how Auger spectroscopy can yield insight into on-site electron correlation, charge transfer and electron screening in a variety of systems and can be used to develop potential models that give rise to a new scale of electronegativity. Studies of particular transitions reveal the local electron configuration and the shape of the local density of states in the vicinity of a core-ionised site. Auger profiles are sensitive to the hybridisation between $d$ bands and $s p$ bands and to the localisation of screening charge. The recent development of high performance instruments and of sophisticated theoretical techniques is allowing Auger spectroscopy to reach its full potential as a probe of local electronic structure.
\end{abstract}

\section{Introduction}

Pierre Auger first detected the radiationless emission of electrons by core-ionised atoms in cloud chamber experiments in 1925 [1]. The recognition that the cloud chamber tracks had an atomic origin and that the energies of the emitted electrons were characteristic of the element established that Auger spectroscopy had the potential to yield information on elemental distributions on an atomic scale. However it has taken a long time to realise the potential of Auger spectroscopy for elemental analysis. For many years following Auger's discovery technical limitations impeded the development of electron spectroscopy and information about Auger processes was principally obtained indirectly through the study of X-rays [2]. Extensive direct studies of Auger spectra only became possible following the development of high resolution electron spectrometers and ultra high vacuum techniques in the 1960's [3,4] and from then on Auger spectroscopy developed alongside X-ray photoelectron spectroscopy (XPS) $[5,6]$.

The most intense Auger transitions involve states in the atomic core and since the spin-orbit coupling constants of core-hole states can be quite large and the electrostatic interactions between core- holes are strong such transitions usually show a pronounced multiplet structure. This term 
structure is well characterised by the $L, S$ and $J$ quantum numbers describing the coupling of the spin and orbital angular momentum of electrons and for deep core-hole states it has a purely atomic origin and is independent of the atomic environment. The kinetic energies of core-corecore (CCC) Auger electrons are differences in the total energies of different core-ionised atomic states and this gives the process its characteristic elemental signature.

The high intensity of CCC processes meant that they were the first to be studied in detail and used in the development of elemental mapping techniques. The Auger decay process is usually independent of the mechanism by which the initial core-hole is created so by using electron excitation it was possible to exploit the ease with which electron beams can be focussed and controlled in order to record the intensity of CCC transitions as a function of the position of the incident electron beam on a specimen. This is the essence of Auger spectroscopy as a method of elemental analysis, a technique which is restricted to the surface region of solids by the short escape depths of low energy electrons. The development of the elemental mapping aspect of Auger spectroscopy, an attempt to realise the full atomic scale potential of Auger's discovery, has made considerable advances and is reviewed in another article in this volume.

This article is concerned with the use of Auger spectroscopy as a probe of local electronic structure. Due to technical limitations this aspect of Auger spectroscopy developed more slowly than the use of Auger spectroscopy as a tool of elemental analysis. To begin with the technique depends on high resolution measurements of Auger lineshapes and the most useful transitions usually involve valence holes in the final state, the core-core-valence (CCV) and core-valence-valence (CVV) processes. These transitions are usually weaker than CCC transitions and are often very much weaker. Moreover since the Auger spectrum is often spread over an energy range comparable to the width of the occupied local density of states, (LDOS) and since the required information on local electronic structure is contained in the fine detail of the Auger lineshape there is very little scope to reduce the resolution of the spectrometer in order to increase its sensitivity. Finally the background of scattered electrons produced when using electron excitation often leads to a signal to background problem. This latter problem can often be solved by employing X-ray excitation and in particular cases by optimising signal to background by exploiting the tunablity of synchrotron radiation to selectively excite the transitions of interest [7].

The key to exploiting the potential of Auger spectroscopy as a probe of electronic structure lies in the development of high performance electron spectrometers which make use of X-ray excitation to reduce the signal to background problem and which are fitted with multidetectors [8-10]. Significant advances have been made in this field in recent years and Auger spectroscopy is beginning to realise its full potential as a probe of local electronic structure [11-15]. However we should note at the outset that in order to make high resolution measurements of the spectra of weak transitions it is often necessary to collect data from a large specimen area, typically $\sim 1 \mathrm{~cm}^{2}$, and this leads to a lack of information concerning specimen homogeneity. By necessity then such studies are usually concerned with establishing the electronic structure, on an atomic scale, of specimens with a high degree of homogeneity. In particular it is difficult to interpret the Auger spectral profiles obtained from elements which occupy more than one local environment in a specimen. This is exactly contrary to the use of Auger spectroscopy for elemental analysis where no information is obtained on electronic structure and priority is given to obtaining information on specimen inhomogeneity on the smallest possible scale. Of course as electron spectroscopies both techniques face the same limitations on the total information that can be obtained from Auger electrons emitted from a surface. They represent opposite extremes of a continuum of choice in instrument design.

In what follows we assume that Auger transitions are well described by a two step process in which the Auger decay of the initial core-hole state can be separated from the creation of the initial core-hole state. Formally this approach is valid provided that the lifetime of the initial 
state is sufficiently long lived and this is usually the case $[16,17]$. We now review in turn the contribution that the Auger spectroscopy of CCC, CCV and CVV transitions can make to the study of local electronic structure. We should note that this information is not usually obtained directly and is often only revealed after a detailed theoretical analysis. In order to keep the review to a manageable length we will ignore molecular systems and concentrate attention on metallic and semiconducting solids.

\section{Core-Core-Core Transitions}

2.1 ENERGY SHIFTS. - While the kinetic energy of an Auger transition is a difference in the total energy of core-ionised atomic states it is useful to represent this difference in terms of the binding energies of the initial and final states so that we may write the Auger kinetic energy for a transition involving the core-levels $a, b$ and $c$ as;

$$
E_{\mathrm{k}}(a b c: L S J)=E_{\mathrm{b}}(a)-E_{\mathrm{b}}(b c ; L S J)
$$

In general the results of atomic structure calculations give good agreement with the experimental Auger energies of free atoms though there are detailed discrepancies which are worthy of further study [18-21]. Relativistic effects become important for heavier elements but are well reproduced by calculations [22-25] and the study of the kinetic energies of KLL Auger processes has provided useful insight into quantum electrodynamic effects [26].

We begin our consideration of how the study of CCC Auger transitions can be used to probe local electronic structure by ignoring the strong $L S J$ term structure of CCC processes since it is rare for this to be modified by the local electronic structure of the atomic environment. Instead we consider the most significant changes induced in CCC transitions by the local electronic structure which are the environmentally determined shifts in the absolute kinetic energies of the transitions, $\Delta E_{\mathrm{k}}$. It is useful to combine a consideration of such shifts in Auger kinetic energies with a study of the corresponding shifts in the binding energies of core-levels measured by XPS, $\Delta E_{\mathrm{b}}$, since the simultaneous analysis of such shifts gives considerably more insight into local electronic structure than can be obtained from an analysis of either contribution separately. This is because there are two contributions to environmentally induced Auger and XPS shifts, an initial state contribution that can be attributed to the dependence of the potential in the core of an atom on the local environment and a final state contribution that arises from the response of the local electronic structure to the creation of core-holes. It is easy to relate $\Delta E_{\mathrm{b}}$ to these two contributions [27];

$$
\Delta E_{\mathrm{b}}=\Delta V-\Delta R
$$

The creation of a double-hole final state makes it slightly more complicated to establish the influence of the two contributions on the kinetic energies of CCC Auger transitions but to a good approximation [27];

$$
\Delta E_{\mathrm{k}} \sim-\Delta V+3 \Delta R
$$

Wagner [28] was the first to exploit the fact that (1) and (2) can be combined to yield a useful quantity which he called the Auger parameter, $\alpha$,

$$
\Delta \alpha=\Delta E_{\mathrm{k}}+\Delta E_{\mathrm{b}} \sim 2 \Delta R
$$

The value of $\Delta \alpha$ is that it involves a difference of kinetic energies and as a result systematic errors in the energy referencing of the spectrometer cancel. By employing X-ray excitation and 
measuring XPS and Auger spectra simultaneously $\Delta \alpha$ can be measured accurately and to a first approximation it gives a direct determination of the environmentally induced differences in the final state screening contribution to the energy associated with the creation of a core-hole [29]. For this reason $\Delta \alpha$ has been termed the final state Auger parameter in recent work [30-32] in order to distinguish it from another way of combining (1) and (2) to form the initial state Auger parameter [30];

$$
\Delta \beta=\Delta E_{\mathrm{k}}+3 \Delta E_{\mathrm{b}} \sim 2 \Delta V
$$

Although systematic errors in energy referencing do not cancel in $\Delta \beta$ this quantity can often be determined accurately and is useful in determining differences in the initial state atomic potential between two environments.

There is considerable interest in separating initial and final state contributions to XPS and CCC Auger energies since these give insight into two important but elusive concepts, charge transfer and electron screening. Thomas and Weightman [33] sought to interpret Auger parameter shifts in terms of a simple model which has frequently be used [4,27,34] to relate the potential in an atomic core to the atomic valence charge;

$$
V=C+k q+U
$$

where $C$ represents the contribution from the nucleus and the core electrons, $k$ is the change in the potential in the core when a valence electron is removed and $U$ is the contribution from the chemical environment. By assuming that $k$ and $q$ depend linearly on the occupancy of coreorbitals, $N$, it can be shown [33] that;

$$
\Delta \alpha=\Delta[q(\mathrm{~d} k / \mathrm{d} N)+(k-2 \mathrm{~d} k / \mathrm{d} N)(\mathrm{d} q / \mathrm{d} N)+(\mathrm{d} U / \mathrm{d} N)]
$$

where the first term represents the relaxation contribution from shrinkage of the occupied valence orbitals when the atom is core-ionised and the second represents the contribution from transfer of screening charge from the surroundings to the core-ionised atom. The third term gives the effect of the polarization of the surroundings by the core-hole.

Equation (7) relates $\Delta \alpha$ directly to differences in valence charge in the ground state, $\Delta q$, and differences in final state screening efficiency $\Delta(\mathrm{d} q / \mathrm{d} N)$. It has been applied to the determination of charge transfer in metal alloys $[33,35,36]$, to the analysis of charge transfer and electron screening in bulk semiconductors $[37,38]$ and at semiconductor surfaces $[30,39]$. The analysis of the second and third terms in (7) is particularly difficult in systems with non-local screening and although current treatments in terms of the Jost cavity model [37, 40] and the polarisable atom model [41, 42] are promising there is a need for theoretical advances in this area if the analysis of Auger parameter shifts is to realise its full potential for studies of delocalised electron screening.

The application of (7) to metallic environments introduces two useful simplifications. Firstly the efficient local screening means that the third term will be zero. Secondly it can be assumed that the core-hole screening is on-site with the implication that $d q / d N$ will be unity with exactly one screening electron attracted from the conduction band to the core-ionised site. For a comparison between two metals $\Delta(\mathrm{d} q / \mathrm{d} N)$ will thus be zero, giving;

$$
\Delta \alpha=\Delta q(\mathrm{~d} k / \mathrm{d} N)
$$

for the case were the valence electrons belong to a single band. When more than one band is involved (7) can be generalised [33] to;

$$
\Delta \alpha=\Delta \Sigma_{i}\left\{q_{i}\left(\mathrm{~d} k_{i} / \mathrm{d} N\right)+\left(\mathrm{d} q_{i} / \mathrm{d} N\right)\left[k_{i}-2\left(\mathrm{~d} k_{i} / \mathrm{d} N\right)\right]\right\}
$$


where the summation is over the partially filled bands. The parameters $k$ and $\mathrm{d} k / \mathrm{d} N$ can be obtained from the results of atomic structure calculations for free atoms [33] corrected in the metal for the effects of the compression of the valence charge which will raise the potential in the atomic core $[33,43]$. The application of (9) to the shifts observed between elemental $\mathrm{Zn}$ and a AuZn alloy and elemental $\mathrm{Au}$ and the AuZn alloy gave consistent results for the magnitude and direction of charge transfer in the alloy. A study of a AuMg alloy was equally successful [33]. A significant contribution to the residual inaccuracy $(\sim 0.03 \mathrm{e})$ of these determinations of charge transfer arises from the difficulty in determining the effects of the compression of the valence electron wavefunctions in the metallic environment. In principle accurate potential parameters can be determined empirically from a comparison of free atom to elemental metal Auger parameter shifts. However when this was attempted [44] for $\mathrm{Na}, \mathrm{Mg}$ and $\mathrm{Zn}$ a contradiction arose between the assumption of perfect local screening in the metal $(\mathrm{d} q / \mathrm{d} N=1)$ and the idea that valence electron compression raises the potential in the atomic cores in the metal. Further analysis by means of Dirac-Fock atomic structure calculations revealed that this contradiction arose from the neglect of significant non-linear terms in the relationship between the valence charge and the atomic potential [44]. For all three elements $k$ was found to depend on the valence charge $q$, a result found earlier by Snyder [45], and on the number of core holes, $N$. Saethre, Siggel and Thomas [46] came to similar conclusions from an analysis of molecular charge distributions. We can thus express $k$ in (6) in the form;

$$
k(N, q)=a+b N+\mathrm{d} q
$$

where $b$ represents the contraction of the valence shell on core-ionization and $d$ arises from the expansion (contraction) of the valence shell of the atom accompanying the gain (loss) of valence charge. Typically it is found that $a \sim 10 \mathrm{eV}, b \sim-2 \mathrm{eV}$ and $d \sim 2 \mathrm{eV}$ with higher order terms an order of magnitude smaller. Inserting the expression for $k(N, q)$ into (6) leads to more complicated expressions for the initial and final state Auger parameter shifts [47];

$$
\begin{aligned}
& \Delta \alpha=\Delta\left\{q b+(\mathrm{d} q / \mathrm{d} N)[a-2 b+d(q-\mathrm{d} q / \mathrm{d} N)]+\mathrm{d} V^{\text {ea }} / \mathrm{d} N\right\} \\
& \Delta \beta=\Delta\left\{2 a q+d q^{2}-(2 / 3)[2 b+d(\mathrm{~d} q / \mathrm{d} N)](\mathrm{d} q / \mathrm{d} N)+2 V^{\text {ea }}\right\}
\end{aligned}
$$

The explicit dependence of the initial state parameter $\Delta \beta$ on the extra-atomic term, $V^{\text {ea }}$, which contains a contribution from the specimen workfunction, explains the sensitivity of this quantity to errors in energy referencing. Such systematic errors cancel in the expression for $\Delta \alpha$ since this depends on $\mathrm{d} V^{\mathrm{ea}} / \mathrm{d} N$. Expressions (11) and (12) have been applied to the free atom to elemental metal Auger parameter shifts for $\mathrm{Na}, \mathrm{Mg}$ and $\mathrm{Zn}$ and the results obtained for the effects of compression of the valence charge, on-site screening of core-holes and the magnitude of valence charges are all consistent with expectations [47].

Recently the parameterised forms of the atomic potentials, (6) and (10), of the first and second row elements have been evaluated and used to derive a new scale of electronegativity [48]. The results are shown in Figure 1 and yield an approximately linear relationship across each period when compared with Pauling's original scale [49]. However the gradients of the lines of comparison with Pauling's scale are significantly different for the first and second periods a result which may be understood in terms of the difference in the atomic radii of first and second row elements [48].

The polynomial expansion of the core potential, (6) and (10), has recently been used to study the contribution that the conduction electrons in a metal make to final state screening [50]. Previously the excited atom model [51, 52], in which the size of extra-atomic screening is evaluated from a neutral core- excited atomic system, had provided quite good agreement with experimental measurements of the strong local screening that occurs in metals. However this latter approach 


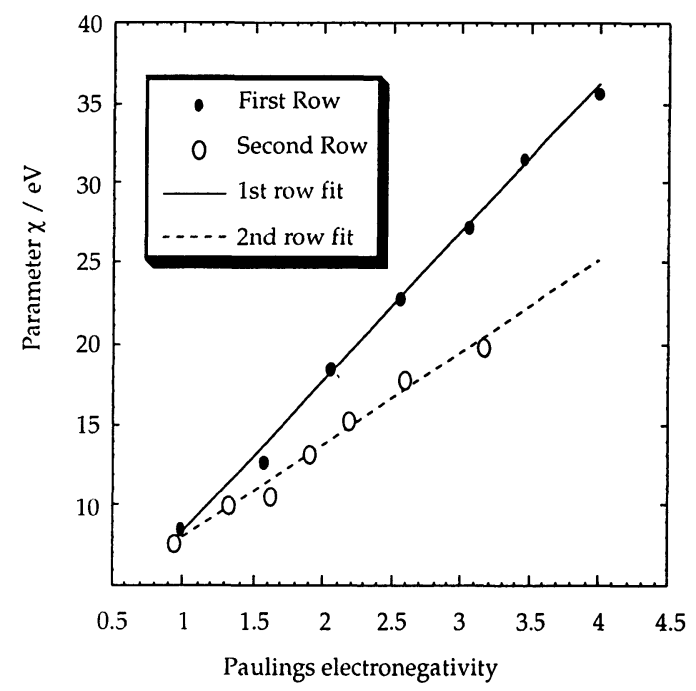

Fig. 1. - A comparison of $\chi$, a parameter derived from the potential model of Jackson, Brooks, Cole and Weightman [48] with Pauling's electronegativity scale.

implies that the details of electron screening in metals are insensitive to the local environment and in consequence that studies of Auger parameter shifts would not give very much insight into the character of screening in different metallic environments. Williams and Lang [52] have provided a sophisticated application of the excited atom model to the calculation of Auger parameter shifts. By extending Williams and Lang's approach [52] to include the effects of the nonlinear dependence of the atomic core potential on the valence charge via (6) and (10) it has been possible to identify the onset of screening by conduction electrons of $d$ character in the alkali and alkaline earths [50]. It is found that the screening in $\mathrm{Na}$ and $\mathrm{Mg}$ is well described by sp screening, that $\mathrm{d}$ screening is necessary to describe the screening of double core-ionised states in the heavier alkali metals and the screening of both single and double core-ionised states in the heavier alkaline earth metals. These results are what one would expect from a simple application of an equivalent cores argument to core-ionised atoms. However the large difference in screening for electrons of $\mathrm{sp}$ and d character demonstrates the potential of measurements of Auger parameter shifts for future studies of electron screening in alloy systems provided the local electronic structure is treated with sufficient sophistication. In this context it would be useful if in the extensive theoretical studies of the electronic structure of metal and alloy systems some attention could be given to the evaluation of atomic core potentials since these are clearly observable quantities that can be measured by a combination of XPS and Auger spectroscopy.

2.2 Multiplet STRUCTURE. - In general the Auger profiles of CCC transitions are dominated by atomic structure effects and a good knowledge of the energies and intensities of the $L S J$ components of the multiplet structure of the corresponding transitions in a free atom makes it possible to identify all the components of the spectral profile observed from the solid state. A theoretical treatment of the Auger spectral profile of a CCC transition requires calculations of the final state multiplet splittings and the transition rate to each component of the multiplet structure. This can usually be done by making use of the results of atomic structure calculations that adopt an independent particle central field approximation.

The final state multiplet structure is determined by the Coulomb repulsion between electrons, 
which can be expressed in terms of Slater $\mathrm{F}^{k}\left(n_{b} l_{b}, n_{c} l_{c}\right)$ and $\mathrm{G}^{k}\left(n_{b} l_{b}, n_{c} l_{c}\right)$ integrals [53], and the spin- orbit coupling $\zeta_{n l}$ L.S. The relative strengths of these two interactions determines the coupling scheme best suited to describing the multiplet structure. The majority of transitions are best described by either a $j j$-LS scheme, in which the initial state is described in $j j$ coupling and the final state in LS coupling, or a $j j$-IC scheme, in which the final state is described by intermediate coupling [54]. This aspect of the multiplet splitting of Auger transitions has been well described previously $[6,11,55,56]$. The spin-orbit coupling constants and Slater integrals can be found from atomic structure calculations. There are differences in detail between the multiplet splittings given by the various theoretical approaches to atomic structure eg; Hartree-Fock (HF), Hartree-FockSlater (HFS), Dirac-Fock (DF) and Dirac-Fock- Slater (DFS), and the experimentally observed splittings. These differences are usually attributed to the neglect of correlation effects within the open shell created by the transition or between configurations of similar binding energy. Tables of the Slater integrals found from a DFS calculation are available for all the elements [57].

In calculating transition rates for an Auger process involving electrons $i, j$ and $k$ in core states $a, b$ and $c$ of a free atom we begin with the Slater determinental wavefunction of the $N$ electron atom, $\Psi(N)$, in which electrons 1 to $N$ occupy single electron orbitals $\phi_{1}$ to $\phi_{N}$. The core-hole created in the initial state of the Auger process is assumed to be long lived so we may generate the wavefunction of the initial state by deleting from the atomic Slater determinant the orbital $\phi_{a}$ and electron $i$ to form $\Psi\left(N-1, \phi_{a}, i\right)$. We expect that the presence of the core hole will cause the single electron orbitals to relax from their form in the ground state giving a new set $\phi_{1}^{*}$ to $\phi_{N}^{*}$. The wavefunction of the final state is given by $\Psi\left(N-1, \phi_{b},\left(\phi_{c} \rightarrow \chi_{d}\right), j\right)$ in which the term in brackets indicates that orbital $\phi_{c}$ has been replaced by the continuum orbital $\chi_{d}$ occupied by the ejected Auger electron. The relaxation in response to the new atomic potential created in the final state will create a new set of single electron orbitals, $\phi_{1}^{* *}$ to $\phi_{N}^{* *}$. The Auger matrix element will be given by;

$$
A_{a b c}=(2 \pi / \hbar)\left|<\Psi\left(N-1, \phi_{a}, i\right)\right| \sum_{p>q}\left(\mathrm{e}^{2} / r_{p q}\right)\left|\Psi\left(N-1, \phi_{b},\left(\phi_{c} \rightarrow \chi_{d}\right), j\right)>\right|^{2}
$$

where the sum over the interelectronic distances, $r_{p q}$, runs over all electrons $p$ and $q$ such that, $p>q$. The differences in the basis sets of single electron orbitals in the initial and final states makes it difficult to evaluate (13) and it is usual to adopt the frozen orbital approximation in which the basis sets in the initial and final states are both considered to be, $\phi_{1}^{*}$ to $\phi_{N}^{*}$. The normalisation and orthogonality properties of the basis set then makes it possible to reduce (13) to an expression made up of direct and exchange integrals involving only those orbitals directly involved in the transition;

$$
A_{a b c}=\left(2 \pi \mathrm{e}^{2} / \hbar\right)\left|<\phi_{a}(1) \chi_{d}(2)\right| 1 / r_{12}\left|\phi_{b}(1) \phi_{c}(2)>-<\phi_{a}(1) \chi_{d}(2)\right| 1 / r_{12}\left|\phi_{b}(2) \phi_{c}(1)>\right|^{2}
$$

Although this expression seems quite straight forward, in general the coupling of the angular momentum of the two particle initial and final states gives rise to quite complex expressions [58, 59] which must be evaluated in order to find the transition rates to each $L S J$ component of the multiplet structure of the final state. For the relatively straight forward case of an atom with completely closed shells except for a single core-hole in the initial state and two holes in the final 
state (14) becomes $[58,60]$;

$$
\begin{aligned}
& A_{a b c}=\left(2 j_{d}+1\right)(2 J+1) \prod_{i=a, b, c, d}\left(2 l_{i}+1\right)(2 L+1)(2 S+1)\left\{\begin{array}{ccc}
1 / 2 & 1 / 2 & S \\
l_{a} & l_{d} & L \\
j_{a} & j_{b} & J
\end{array}\right\} \\
& \times \mid \sum_{K}\left(\begin{array}{ccc}
l_{a} & k & l_{b} \\
0 & 0 & 0
\end{array}\right)\left(\begin{array}{ccc}
l_{d} & k & l_{c} \\
0 & 0 & 0
\end{array}\right)\left(\begin{array}{ccc}
l_{a} & l_{b} & k \\
l_{c} & l_{d} & L
\end{array}\right) D\left(k l_{d}\right) \\
& +\left.(-1)^{L+S} \sum_{K}\left(\begin{array}{ccc}
l_{a} & k & l_{b} \\
0 & 0 & 0
\end{array}\right)\left(\begin{array}{ccc}
l_{d} & k & l_{c} \\
0 & 0 & 0
\end{array}\right)\left(\begin{array}{lll}
l_{a} & l_{b} & k \\
l_{c} & l_{d} & L
\end{array}\right) E\left(k l_{d}\right)\right|^{2}
\end{aligned}
$$

with

$$
\begin{aligned}
& D\left(k, l_{d}\right)=\iint R_{a}\left(r_{1}\right) R_{d}\left(r_{2}\right)\left(r_{<}^{k} / r_{>}^{k+1}\right) R_{b}\left(r_{1}\right) R_{c}\left(r_{2}\right) \mathrm{d} r_{1} \mathrm{~d} r_{2} \\
& E\left(k, l_{d}\right)=\iint R_{a}\left(r_{1}\right) R_{d}\left(r_{2}\right)\left(r_{<}^{k} / r_{>}^{k+1}\right) R_{b}\left(r_{2}\right) R_{c}\left(r_{1}\right) \mathrm{d} r_{1} \mathrm{~d} r_{2}
\end{aligned}
$$

In order to evaluate these expressions it is also necessary to know the value of the relevant radial matrix elements $D\left(k, l_{d}\right)$ and $E\left(k, l_{d}\right)$. Fortunately comprehensive tables of the latter have been produced by McGuire [58 and references therein] who adopts an independent particle, central field, frozen orbital approach with radial wavefunctions, $R_{n l}(r)$, taken from an HFS treatment of atomic structure [61]. Extensive calculations of transition rates within a relativistic treatment of atomic structure have been published by Crasemann, Chen and co-workers [62-66] though the radial matrix elements necessary to apply this approach the calculation of the intensities of individual $L S J$ components of the multiplet structure are not generally available.

An example of the current state of our understanding the atomic spectra of free atoms is given by Vayrynen et al. [67] analysis of the spectra of the $\mathrm{M}_{45} \mathrm{~N}_{45} \mathrm{~N}_{45}$ Auger transitions of free $\mathrm{Ag}$ atoms (Fig. 2). The final states of these transitions can also be observed in the optical spectra of $\mathrm{Ag}$ and this helps in the interpretation of the multiplet splittings. Vayrynen et al. [67] find small discrepancies between the observed multiplet structure and theoretical results for the splittings and intensities of the multiplet structure components obtained from atomic structure calculations. However these discrepancies are not sufficient to cast doubt on the identification of $L S J$ components and can be attributed to the effects of electron correlation.

Figure 3 shows a comparison of Aksela et al. results [68] for the $\mathrm{M}_{45} \mathrm{~N}_{45} \mathrm{~N}_{45}$ Auger spectra of free atom and metallic Cd. The metallic spectrum is shifted $\sim 12 \mathrm{eV}$ to higher kinetic energy as a result of electron screening in the final state $[23,68]$. Theoretical calculations of the multiplet structure show the same degree of agreement with experiment as is obtained for the spectra of the same transitions for atomic $\mathrm{Ag}[23,67,68]$. A comparison of the free atom and metallic spectra show that the multiplet structure is preserved in the metallic state though with an increase in the linewidth of $L S J$ components. Such an increase is observed in the "quasi atomic" spectra observed from other metals $[23,69]$. In Cd the component linewidth increases from $\sim 0.3 \mathrm{eV}$ in the free atom to $\sim 0.8 \mathrm{eV}$ in the metal [68]. The lifetime broadening of components of the multiplet structure is given by the sum of the lifetime broadenings of the initial core-hole state and the two hole final state. The final states created in atomic $\mathrm{Cd}$ by these transitions belong to the $4 d^{8} 5 s^{2}$ configuration and these are particularly long lived since they cannot decay by the emission of dipole radiation or by further Auger emission. It is reasonable to expect that these states will experience an increased lifetime broadening in the metal due the occurance of decay processes not present in the free atom such as Auger processes made energetically possible by the broadening of the $\mathrm{d}$ and $\mathrm{sp}$ band. 


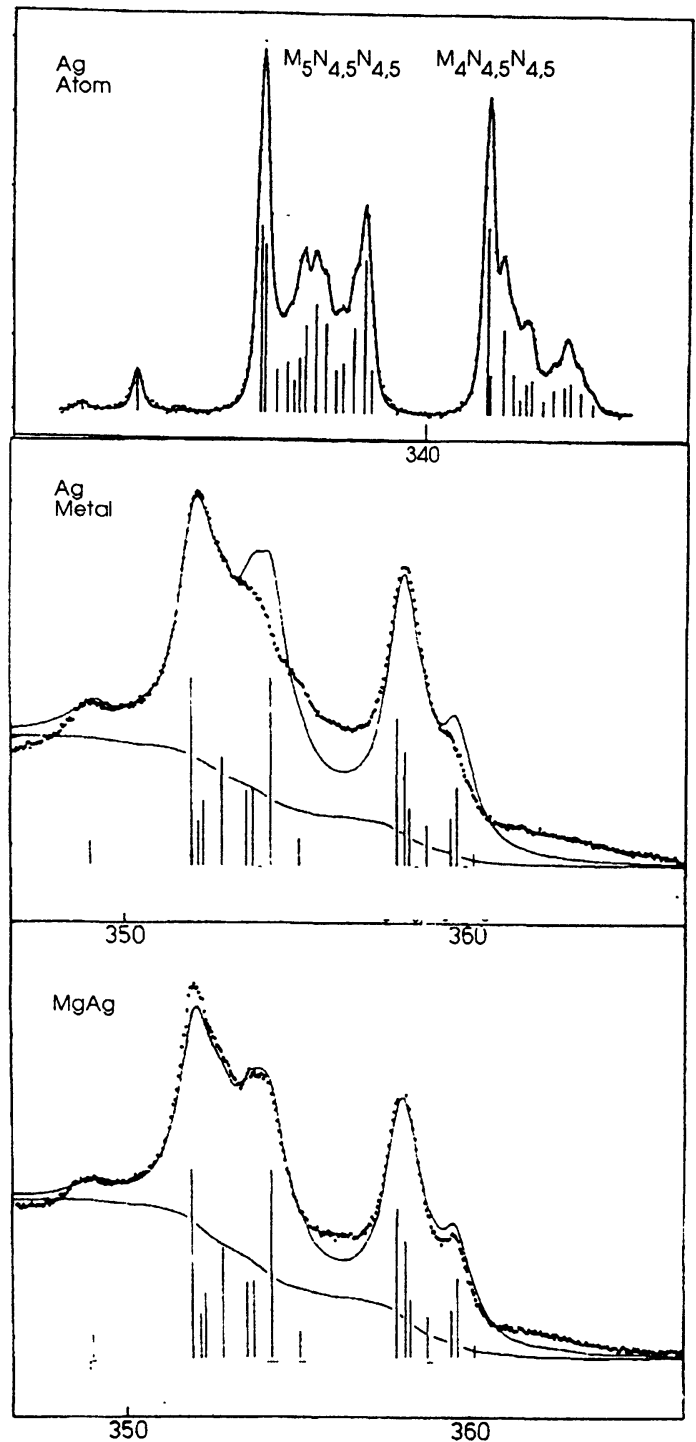

Fig. 2. - The top panel shows the spectral profile of the $\mathrm{M}_{4,5} \mathrm{~N}_{4,5} \mathrm{~N}_{4,5}$ transitions of atomic $\mathrm{Ag}$ observed by Vayrynen $e t$ al. [67]. The middle and bottom panels show the spectral profiles of the $\mathbf{M}_{4,5} \mathrm{~N}_{4,5} \mathrm{~N}_{4,5}$ transitions of $\mathrm{Ag}$ in metallic $\mathrm{Ag}$ and $\mathrm{MgAg}$ respectively measured by Weightman et al. [123].

\section{Auger Transitions Involving Valence Levels}

In the previous section we saw how the frozen orbital approximation makes it is possible to simplify Slater determinental atomic wavefunctions and reduce the Auger matrix element to an expression involving only those orbitals directly involved in the transition. The assumption that the wavefunctions of states occupied by electrons not directly involved in an Auger transition do not change as a result of the transition is not too unreasonable for CCC transitions since atomic core states experience a strong atomic core potential due to the nucleus and strong electron-electron 

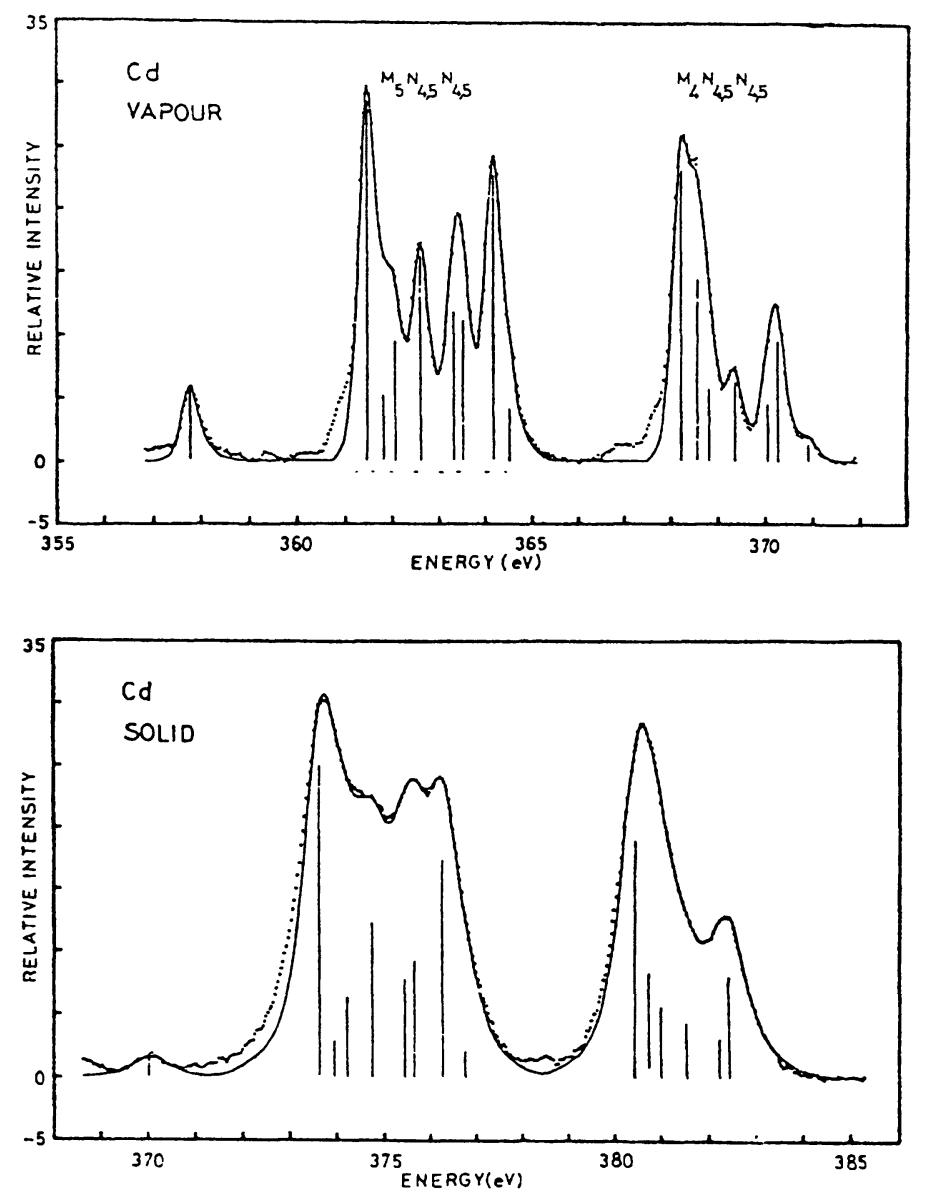

Fig. 3. - The spectral profiles of the $\mathrm{M}_{4,5} \mathrm{~N}_{4,5} \mathrm{~N}_{4,5}$ transitions of atomic and metallic Cd measured by Aksela et al. [68].

interactions. However when the Auger process creates a hole state in a valence level it is likely that the wavefunctions of the weakly bound valence electrons respond strongly to the difference in atomic potential between the initial and final states involved in the transition. These considerations are particularly important for Auger processes involving the conduction electrons of a metal since these are free to respond to the change in potential and we might expect the wavefunctions of the states occupied by conduction electrons in the initial and final states to be significantly different. In order to take account of these changes we require a more sophisticated analysis of the Auger matrix element using wavefunctions described by Slater determinants of valence states that are different in the initial and final states. Fortunately this problem has been addressed by a several theoreticians [70-74] who have collectively established the validity of the "final state rule" to describe transitions for which it is possible to neglect on-site electron correlation, a restriction which essentially limits the application of the rule to transitions not involving $d$ electrons. The final state rule states that for Auger transitions which create final hole states in the conduction band of metals;

a) The shape, or energy dependence, of the conduction band s and p contributions to the Auger 
profile is given by the shape of the LDOS in the final state.

b) The intensity of the conduction band $s$ and $p$ contributions to the Auger profile is given by the local electron configuration in the initial state.

The implication of the final state rule is that the spectra of Auger transitions involving valence levels contain information on the shape of the local screening density of states together with its orbital character. We will now examine the progress made in extracting this information from the CVV and CCV Auger spectra of metallic systems.

3.1 Core-VAlenCe-VAlenCE TRAnsitions. - We begin by considering the CVV spectra of simple metals. By combining measurements of Auger spectra with XPS measurements of the binding energy of the initial state and referencing both to a common reference energy such as the Fermi energy we can deduce from (1) the experimental profile of the density of double-hole states;

$$
E_{\mathrm{b}}(\mathrm{VV})=E_{\mathrm{b}}(\mathrm{C})-E_{\mathrm{k}}(\mathrm{CVV})
$$

where we have dropped the explicit dependence on the $L S J$ multiplet structure of the two hole final state. These angular momentum considerations are important and they have a strong influence on the shape of the Auger profile. However the description of final state components in terms of the $L S J$ term structure is only appropriate when the local atomic potential is strong enough to dominate the crystal potential and in consequence the term structure appropriate to localised atomic states does not appear in the spectra observed from transitions which create final states in the delocalised conduction band states of simple metals.

The final state rule leads us to expect that the profile of CVV transitions contains information on the energy dependance of the local density of states in the two-hole final state and the orbital character of the valence electrons in the initial, screened, core-ionised state. Ideally such profiles should be used to check the predictions of full self-consistent field calculations of the local electronic structure in which the Auger matrix elements and their energy dependance across the conduction band are evaluated. This is a difficult theoretical task since the core-hole potential in the initial state breaks the translational symmetry that is the basis of most band structure calculations [15]. Instead various approximations are made, usually in the context of the final state rule. An important simplification for simple metals is to assume that since the changes in local electronic structure due to the creation of holes in the delocalised states of the conduction band is likely to be weak then these states will be well represented by the local electronic structure in the ground state. It is then only necessary to map the results of band structure calculations on to a local basis set expressed in terms of the angular momentum of the conduction band states and account for Auger matrix element effects. The angular momentum character of the final hole states created in a CVV transition has a strong influence on the spectral profile via the matrix elements which are combinations of Clebsch-Gordan angular momentum coupling coefficients and exchange and Coulomb radial integrals. If we assume that the radial integrals can be decoupled from the energy dependence of the LDOS then, restricting the analysis to states of $s$ and $p$ valence character, we can express the contribution of the double hole density of states to an Auger profile as $[75,76]$;

$$
\begin{aligned}
E_{\mathrm{b}}(\mathrm{VV})= & {[x a]^{2} \cdot D_{\mathrm{s}}(E) * D_{\mathrm{s}}(E) \cdot M_{\mathrm{Css}}^{2}+[x a y b] \cdot D_{\mathrm{s}}(E) * D_{\mathrm{p}}(E) \cdot M_{\mathrm{Csp}}^{2}+} \\
& {[b y]^{2} D_{\mathrm{p}}(E) * D_{\mathrm{p}}(E) \cdot M_{\mathrm{Cpp}}^{2} }
\end{aligned}
$$

where in the initial state the core-ionised atomic core has a screening configuration $n \mathrm{~s}^{x} n \mathrm{p}^{y}$ and the final state configuration may be approximated by a ground state configuration $n \mathrm{~s}^{a} n \mathrm{p}^{b} . D_{\mathrm{s}}(E)$ and $D_{\mathrm{p}}(E)$, which appear in (17) as convolutions, represent the LDOS of $s$ and $\mathrm{p}$ character, normalised to unity and the $M_{\mathrm{Cl}}$ are the energy independent radial and angular parts of the matrix elements. 
In order to relate the results of the theoretical expression (17) to observed CVV profiles it is also necessary to allow for instrumental effects and for the background of scattered electrons. The former should be well known and while the latter can rarely be calculated from first principles it usually has only a small influence on the observed spectral profile and its character is sufficiently understood for it to be modelled successfully [77]. Provided the local densities of states and the matrix elements can be determined it is then possible to deduce the relationship between the ground state and screening configurations in a simple metal by comparing the profile deduced from (17) with experiment. One approach is to determine the local ground state densities of states from band structure calculations evaluated in terms of a local atomic orbital basis set and make use of atomic matrix elements obtained from atomic structure calculations for the corresponding transitions in free atoms [78]. The latter approximation, while not ideal, is quite reasonable when it is remembered that the presence in the Auger matrix element of the wavefunction of the initial core-hole state will ensure that the only parts of the radial wavefunctions of valence orbitals that contribute to the transition rate will be those in the core region. Furthermore the requirement that the valence orbitals be orthogonal to the core- orbitals means that the core region of the valence orbitals will not be strongly perturbed by the solid state environment and finally the presence of the core-hole in the initial state will deepen the potential on the site and draw in the valence wavefunctions weakening the influence of the solid state potential.

The analysis set out in the previous paragraph makes clear that it is possible to obtain useful information from CVV spectra on the changes in local configuration induced by a core-hole though extracting this information requires high quality experimental results together with a sophisticated theoretical analysis. The potential of this approach can be seen from studies of the $\mathrm{L}_{2,3} \mathrm{VV}$ and $\mathrm{KVV}$ spectra of $\mathrm{Li}, \mathrm{Be}$ [79] and $\mathrm{Mg}$ [76] metals. The study of Be [79], for example, established that whereas the ground state configuration, deduced from band structure calculations, is $1 s^{2} 2 s^{0.68} 2 p^{1.32}$ the initial state of the Auger transition is screened by electrons of $s$ character yielding a local configuration for the core ionised site of $1 \mathrm{~s}^{2} 2 \mathrm{~s}^{1.68} 2 \mathrm{p}^{1.32}$.

Almbladh and co-workers $[80,81]$ have calculated CVV profiles for the LVV and KVV transitions of a number of simple metals within a sophisticated theoretical treatment which includes many electron effects. It is clear from these calculations that the Auger profiles of CVV processes in simple metals can be used to assess the importance of many electron effects in these materials. Unfortunately there are still only a few high resolution measurements of experimental profiles with which to compare the theoretical results. The transition rates of KVV processes for the second row elements are particularly weak and high resolution data can only be obtained with the most sensitive of spectrometers $[10,76]$. Recently high resolution spectra have been obtained for the KVV transitions of Si [10] and an analysis of these results has yielded a useful insight into the local electronic structure in the ground state of this important material [82].

3.2 Core-VAlence-Valence Transitions; Electron Correlation. - An important restriction on the final state rule is that it only applies to CVV transitions for which the electron correlation, $U$, between the two holes created in the final state can be neglected. As $U$ increases the rule ceases to apply and in the limit of large $U$ one observes [83] a "quasi-atomic" profile which is unrelated to the LDOS in the solid and which contains all the $L S J$ components of the multiplet structure that are present in the spectrum of the free atom. We noted this phenomena earlier for the $\mathrm{M}_{45} \mathrm{~N}_{45} \mathrm{~N}_{45}$ transitions of metallic Cd (Fig. 3). $U$ is usually only significant for final states created in the localised orbitals of $d$ electrons and for this reason quasi-atomic profiles are generally observed for Auger transitions that create two-hole final states in d bands. Such Cdd transitions may be regarded as intermediate between the CCC transitions which involve only core-hole states, and which show $L S J$ multiplet splittings that are independent of the atomic environment, and CVV transitions which are strongly influenced by the LDOS of the valence electrons. The 
spectral profiles of such transitions thus contain information on the electron correlation between the final hole states and the extent of their localisation on the atom. The latter attribute governs the width, $W$, of the single electron DOS which can be measured by XPS. The value of $U$ and its relationship to $W$ are important characteristics of the electronic structure of molecules and solids that can be uniquely studied by an analysis of Auger profiles. The explanation of the origin of quasi-atomic Auger spectra was achieved in independent formulations by Cini [84, 85] and Sawatzky [86] of a theory relating the CVV profile to the ratio of the on-site electron correlation $U$ to the single electron bandwidth $W$. The theory shows how the profile depends on the ratio of $U / W$. For $U \sim 0$ the profile is indistinguishable from a self-convolution of the $\operatorname{LDOS}, D^{0}(E)$. As $U$ increases the profile shows an increase in the intensity in the most bound region of $D^{0}(E)$ which develops into a sharp peak. As $U$ increases beyond the value of $W$ a sudden transition occurs to a state in which the majority of the intensity is contained in sharp localised state which splits off to higher binding energy of $D^{0}(E)$. As $U / W$ increases further the split off, "quasi-atomic", peak rapidly accounts for a majority of the intensity in the transition with the remainder distributed in a broad residual "quasi-band" like component in the energy range of $D^{0}(E)$. By recognising that $U$ is different for each $L S J$ component of the final state multiplet structure Weightman and Andrews [9] established the essence of the Cini-Sawatzky theory by monitoring the changes in the contribution of individual $L S J$ components to the overall profile of the $\mathrm{M}_{45} \mathrm{~N}_{45} \mathrm{~N}_{45}$ Auger spectra of $\mathrm{Ag}$ as a function of $U(L S J) / W$ as the width, $W$, of the $\mathrm{Ag} \mathrm{d}$ band was varied by alloying $\mathrm{Ag}$ with the simple metals $\mathrm{Mg}$ and $\mathrm{Al}$.

According to Cini-Sawatzky theory the Auger profile of transitions which create two hole final states in a band of width $W$ is given by [14];

$$
A_{\mathrm{Cdd}}(E)=\sum_{L S J} I(L S J) D^{0}(E) /\left\{[1-U(L S J) H(E)]^{2}+\pi^{2} U^{2}(L S J) D^{0}(E)^{2}\right\}
$$

where $I(L S J)$ is the transition rate to the $L S J$ component of the multiplet structure, $U(L S J)$ is the on-site electron Coulomb repulsion between electrons localised in the $L S J$ component of the multiplet structure, and $H(E)$ is the Hilbert transform of $D^{0}(E)$. (18) leads us to expect that the Auger profile will be an envelope of the contributions from the different $L S J$ components of the multiplet structure the lineshapes of each of which depend on the particular value of the ratio $U(L S J) / W$.

In Figure 4 we show the evolution, in accordance with (18), of the Auger profile of an idealised representation of the $\mathrm{M}_{5} \mathrm{~N}_{45} \mathrm{~N}_{45}$ transitions of $\mathrm{Ag}$ as the values of the $U(L S J)$ increase and $W$ remains constant. In the calculation the $L S J$ components of the multiplet structure of the $4 \mathrm{~d}^{8}$ final states created by these transitions have been assigned the intensities and energies given by atomic structure calculations, the splittings being expressed relative to the energy of the most intense component, $U\left({ }^{1} \mathrm{G}_{4}\right)$, which is the only free parameter in the calculation. The small splittings introduced by interactions with the $5 \mathrm{~s}$ electrons, which can just be discerned in the high resolution spectra of free atoms [67] (Fig. 2), are neglected. In these calculations the $\mathrm{Ag} \mathrm{d}$ band is represented by a $3.5 \mathrm{eV}$ wide square band the self-convolution of which, $D^{0}(E)$, gives rise to a triangular profile with a base width of $7.0 \mathrm{eV}$. A component with $U(L S J)=0$ will give rise to a triangular profile with intensity $I(L S J)$. As $U$ increases the profile will be distorted. The top panel of Figure 4 corresponds to a value of $U\left({ }^{1} \mathrm{G}_{4}\right)$ which is too small to yield $U(L S J) / W$ ratios large enough to create split off states. As a result the $M_{5} N_{45} N_{45}$ profile will consist of an envelope of lineshapes with varying degrees of distortion. In the next panel $U\left({ }^{1} \mathrm{G}_{4}\right)$ has been increased to the point where the ${ }^{1} \mathrm{~S}_{0}$ component has split off to form a bound state and several of the other $L S J$ components are close to forming bound states. In the next panel $U\left({ }^{1} \mathrm{G}_{4}\right)$ is high enough for all but the least bound states, ${ }^{3} \mathrm{~F}_{2,3,4}$, to have split off from $D^{0}(E)$. It may be noted that although 
several components of the $L S J$ multiplet structure are split off from $D^{0}(E)$ in this figure their positions are still strongly influenced by (18) and as a result the separation of $L S J$ components is strongly distorted from those expected for a free atom. In the final panel $U\left({ }^{1} \mathrm{G}_{4}\right)$ is sufficiently high for all the $L S J$ components to form bound states, the quasi band features have negligible intensity and the $L S J$ multiplet structure is that of the free atom.

In the experiments on MgAg and AlAg alloys it was found [9] that the $U(L S J)$ are essentially independent of the alloy but that the $\mathrm{d}$ band width, $W$, was sensitive to alloy composition. A comparison of the $\mathrm{M}_{45} \mathrm{~N}_{45} \mathrm{~N}_{45}$ Auger profile as a function of alloy composition thus provided a test of (18) in which the $U(L S J)$ are approximately constant and $W$ varies. Thus for pure $\mathrm{Ag}$ with a d band width of $\sim 3.5 \mathrm{eV}$ the $U(L S J) / W$ ratio for the least bound components, ${ }^{3} \mathrm{~F}_{2,3,4}$, is insufficient to produce split-off states and the spectral profile departs from that expected from an atomic profile (Fig. 2). Reducing the $\mathrm{Ag} \mathrm{d}$ band width to $\sim 2 \mathrm{eV}$ in $\mathrm{MgAg}$ is sufficient to create bound states for all $L S J$ components and the spectrum has a quasi-atomic profile (Fig. 2).

Equation (18) says nothing about the shape of the individual $L S J$ components which, apart from the distortion given by $U(L S J) / W$, we expect to have a Lorentzian lineshape with a width given by the sum of the lifetime broadening of the single core hole initial state and double hole final state. In the Sawatzky [86] formulation of the problem one contribution to the lifetime broadening of the final state can be estimated from the timescale for correlated hopping of the two-hole states $[86,87]$ though other contributions to lifetime broadening are probably of more significance. Equation (18) is able to explain the general variation with $U(L S J) / W$ of the $\mathbf{M}_{45} \mathbf{N}_{45} \mathbf{N}_{45}$ Auger profiles of $\mathrm{Cd}, \mathrm{Ag}$ and $\mathrm{Pd}$ and their alloys with simple metals and an analysis of the spectral profiles yields the values of the on-site electron correlation energies, $U(L S J)$, for these systems. For alloys containing more than one $\mathrm{d}$ band element the mixing between the $\mathrm{d}$ bands can have a radical effect on the Auger profile [88, 89] and equation (18) has been able to explain these profiles in detail for the CdAg [90], AgPd [91] and CuPd [89] alloy systems in terms of theoretical values of the appropriate $D^{0}(E)$. For the CuPd systems the analysis of the Pd Auger profile has made a crucial contribution to the controversy $[35,89,92,93]$ over the extent of $d$ band mixing and the shape of the local Pd density of states in this alloy system. For this and other Pd alloys Auger studies [14] have established that the $U(L S J)$ of $\mathrm{Pd}$ are essentially atomic properties with $U\left({ }^{1} \mathrm{G}_{4}\right) \sim$ $3.0 \pm 0.2 \mathrm{eV}$ for $\mathrm{Al}_{80} \mathrm{Pd}_{20}, \mathrm{Mg}_{75} \mathrm{Pd}_{25}, \mathrm{Cu}_{95} \mathrm{Pd}_{5}, \mathrm{Ag}_{80} \mathrm{Pd}_{20}$ and $\mathrm{Ag}_{95} \mathrm{Pd}_{5}$. This result is in keeping with Hubbard's original formulation of on-site electron correlation but is interesting contrast with recent work for $\mathrm{Cu}$ alloys which has shown that whereas $U\left({ }^{1} \mathrm{G}_{4}\right)$ is similar for $\mathrm{Cu}$ in the metallic element and $\mathrm{Mg}_{2} \mathrm{Cu}$, with values of 6.6 and $6.2 \mathrm{eV}$ respectively, this quantity is larger, $8.1 \mathrm{eV}$, in $\mathrm{Cu}_{50} \mathrm{Pd}_{50}$ [94].

The Cini-Sawatzky theory of Cdd Auger transitions is only valid for systems with filled bands. For systems with incompletely filled bands a significant density of states at the Fermi energy introduces additional correlations which are difficult to evaluate. It is expected that these additional correlations will not have a strong effect for the wide bands of simple metals [95-7] but will be important for systems involving transition metals. Some progress has been made in extending the theory to systems in which the $d$ band is almost full [96-98] but we do not have a theory which would make it possible to deduce values of $U(L S J)$ from the profiles of Auger transitions which create two-hole states in the $\mathrm{d}$ bands of transition metals. Such a theory would be importan $\_$since it would open up the prospect of establishing Auger spectroscopy as a probe of those aspects of electron correlation which play an important role in the electronic structure of transition metals particularly with respect to magnetic properties.

We would expect that the spectral profiles of Auger transitions which create double hole states in unfilled $d$ bands would show a multiplet structure which is very sensitive to the number of $d$ electrons and experimentally this is found to be true for the spectra observed from atomic Fe, Co and $\mathrm{Ni}$ [99]. Rather surprisingly however the spectral profiles of Cdd transitions observed from 


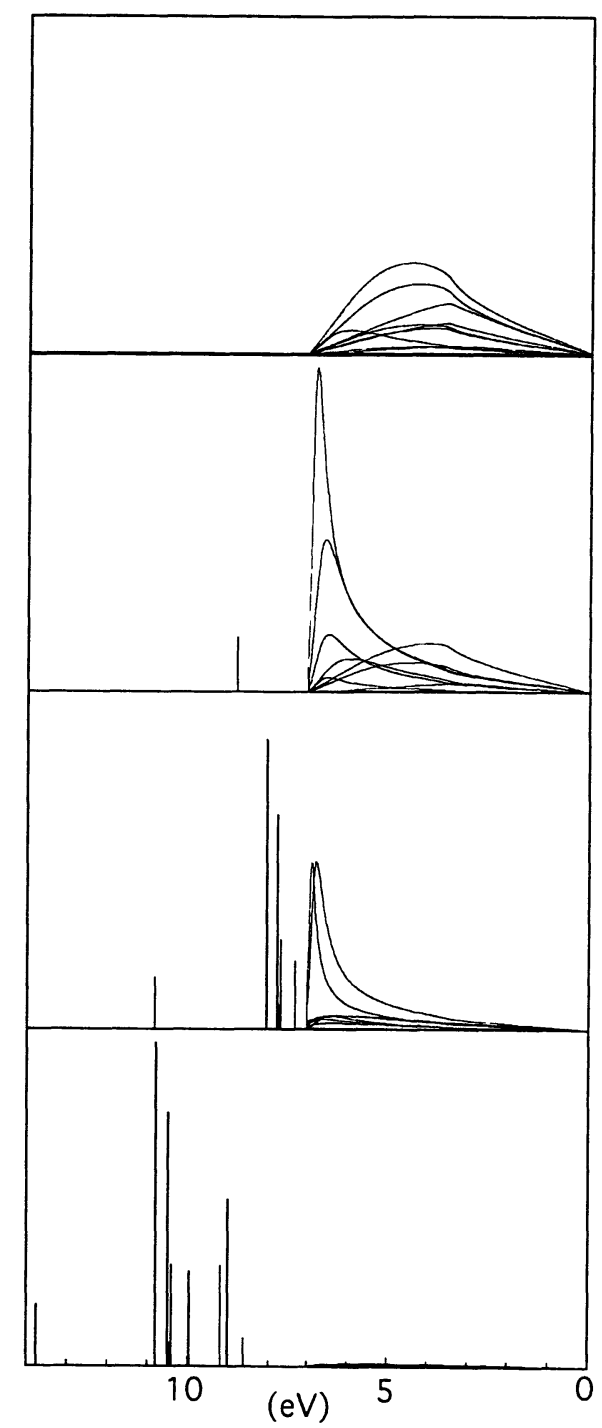

Fig. 4. - This shows the evolution, in accordance with (18), of the Auger profile of an idealised representation of the $\mathrm{M}_{5} \mathrm{~N}_{4,5} \mathrm{~N}_{4,5}$ transitions of $\mathrm{Ag}$ as the values of $U(L S J)$ increase and $W$ remains constant at 3.5 $\mathrm{eV}$. Aside from the calculation shown in the top panel the splittings and intensities of the multiplet structure components have been assigned the values found for free Ag atoms in [104] and the splittings of $L S J$ components are expressed relative to the value of $U\left({ }^{1} \mathrm{G}_{4}\right)$ which is the only free parameter in the calculation. The lower three panels correspond to values of $U\left({ }^{1} \mathrm{G}_{4}\right)$ of $7.0 \mathrm{eV}, 4.0 \mathrm{eV}$ and $2.4 \mathrm{eV}$ in ascending order respectively. The upper panel corresponds to a calculation in which $U\left({ }^{1} \mathrm{G}_{4}\right)$ is set at $2.4 \mathrm{eV}$ but the splittings have been reduced by $60 \%$ from the atomic values in order to avoid the creation of negative values for the least bound components. The figure was calculated by Verdozzi and is unpublished.

the metallic form of the $3 \mathrm{~d}$ transition elements are remarkably similar [100]. It is possible that this similarity may arise from the ability of screening electrons of $d$ character to reduce the values of the $\mathrm{F}^{2}(\mathrm{~d}, \mathrm{~d})$ and $\mathrm{F}^{4}(\mathrm{~d}, \mathrm{~d})$ integrals responsible for the splitting of $L S J$ components and thus 
"collapse" the multiplet structure. There is some evidence that this happens for Pd alloys [89].

Although it has been shown for the $\mathrm{M}_{45} \mathrm{~N}_{45} \mathrm{~N}_{45}$ transitions of $\mathrm{Ag}$ and its alloys with $\mathrm{Mg}$ and $\mathrm{Al}$ [9] that Cini-Sawatzky theory gives a detailed explanation of the variation in the relative intensity of multiplet structure components as a function of $U(L S J) / W$ this and similar studies have not established that the theory can account simultaneously for the dependence of both the kinetic energy and the profile of Auger spectra on $U(L S J) / W$. In fact a study of the $\mathrm{N}_{6,7} \mathrm{O}_{4,5} \mathrm{O}_{4,5}$ Auger transitions of metallic $\mathrm{Au}$ [101] has shown that Cini-Sawatzky theory does not give simultaneous agreement with the kinetic energy and profile of these transitions. This failure may be traced to the treatment of electron correlation in terms of the on-site interaction of the Hubbard Hamiltonian and it has been shown $[102,103]$ that Auger profiles are sensitive to the spatial dependence of the electron correlation interaction. The inclusion of a dependence on the distance, $R$, between the final state holes in the electron correlation term, $U(L S J, R)$ results in a contribution from off-site correlations which effectively adds an additional self-energy correction term to (18). This term introduces a shift in the binding energy of the split off states and this shift of $\sim 1.2 \mathrm{eV}$ is able to resolve the discrepancy observed between Cini-Sawatzky theory and the experimental results for the $\mathrm{N}_{67} \mathrm{O}_{45} \mathrm{O}_{45}$ transitions of Au. Recently [104] the $\mathrm{M}_{45} \mathrm{~N}_{45} \mathrm{~N}_{45}$ Auger spectral profile of metallic $\mathrm{Ag}$ has been reassessed in the light of the extension of Cini-Sawatzky theory to include off-site interactions and they are found to generate a larger shift in $\mathrm{Ag}, 2.3 \mathrm{eV}$, to that observed for Au. Since the Hubbard Hamiltonian is central to the study of strongly correlated systems exhibiting magnetism and high temperature superconductivity the recent Auger studies extending the two particle Hubbard Hamiltonian to include off-site interactions is likely to prove a significant development. The electron screening in these oxide materials is expected to be much less efficient than in normal metals and this should increase the strength of the off-site contribution to the Hubbard Hamiltonian.

3.3 CORE-CORE-VALENCE TRANSITIONS. - Historically attempts to interpret anomalies in the CCV spectra of simple metals played an important role in establishing the final state rule. The key development was the theoretical study of the KLV spectra of metallic $\mathrm{Na}$ by von Barth and Grossman [105]. Since $\mathrm{Na}$ is a good free electron metal it was expected that the spectral profiles of KLV transitions, which create a single final state hole in the conduction band, should reflect the LDOS of the conduction band modified by the influence of matrix element effects. The conduction band densities of states have been observed directly by XPS for the simple metals, Na, $\mathrm{Mg}$ and $\mathrm{Al}$, and these follow the shape expected from the Fermi distribution [106, 107]. However although the $\mathrm{KL}_{2,3} \mathrm{~V}$ profiles could be interpreted in terms of a Fermi distribution the spectra of the $\mathrm{KL}_{1} \mathrm{~V}$ transitions showed a puzzling double peaked structure $[108,109]$ that was also observed in the corresponding transitions of metallic $\mathrm{Mg}$ and $\mathrm{Al}$. Since the $\mathrm{L}_{1}$ and $\mathrm{L}_{2,3}$ final states involve core-hole states, which XPS showed to be quite narrow and therefore relatively long lived, it was expected that they should only introduce a small, $\sim 1 \mathrm{eV}$, lifetime broadening of the KLV profiles and could not account for the two peaked structure of the $\mathrm{KL}_{1} \mathrm{~V}$ profile. However von Barth and Grossman [105] recognized that the presence of a core-hole in the final state would lead to a local distortion of the conduction band density of states and that the spectral shapes of CCV transitions would be determined by the LDOS of this core-ionised final state. Detailed calculations for $\mathrm{Na}$ showed that the LDOS of s character, $D_{\mathrm{s}}^{1}(E)$, was strongly distorted by the presence of a core-hole creating a peak at the bottom of the conduction band. The shape of the LDOS of $\mathrm{p}$ character, $D_{\mathrm{p}}^{1}(E)$, was found to be relatively insensitive to the presence of a core-hole though the magnitude of $D_{\mathrm{p}}^{1}(E)$ did increase to reflect the increased screening of the core-hole. An analysis of KLV matrix elements shows that the $\mathrm{KL}_{2,3} \mathrm{~V}$ lineshape will be dominated by $D_{\mathrm{p}}^{1}(E)$ with only a small contribution from $D_{\mathrm{s}}^{1}(E)$ while the $\mathrm{KL}_{1} \mathrm{~V}$ lineshape will receive approximately equal contribu- 
tions from $D_{\mathrm{s}}^{1}(E)$ and $D_{\mathrm{p}}^{1}(E)$ and thus show a double peak. Von Barth and Grossman's analysis [105] provided a good understanding of the KLV spectra of $\mathrm{Na}$ which has since been confirmed by detailed theoretical studies for the simple metals $\mathrm{Mg}$ and $\mathrm{Al}$ [110-112].

Unfortunately first principles approaches to the interpretation of CCV profiles are difficult and rare since they require self consistent field calculations of the density of states in the coreionised final state in which the translational symmetry, which expedites efficient band structure calculations, is broken by the presence of the core-hole in the final state [15]. However combining the ideas which lead to the representation of the profile of CVV transitions in terms of (17) with the final state rule, suggests that the profile of CCV transitions for systems with $s$ and $p$ valence states can be expressed in a simple form;

$$
E_{\mathrm{b}}(\mathrm{CV})=M_{\mathrm{CCs}}^{2} D_{\mathrm{s}}^{1}(E)+M_{\mathrm{CCp}}^{2} D_{\mathrm{p}}^{1}(E)
$$

where $D_{\mathrm{s}}^{1}(E)$ and $D_{\mathrm{p}}^{1}(E)$ are unnormalised and implicitly include the amount of $\mathrm{s}$ and $\mathrm{p}$ character in the LDOS in the presence of a single core-hole. $M_{\mathrm{CCs}}$ and $M_{\mathrm{CCp}}$ are the appropriate matrix elements. Equation (19) is based on the assumptions that the matrix elements are independent of both the Auger energy and the energy of the valence electrons and that the full expression for the Auger profile can be separated into LDOS and matrix element components. Equation (19) is useful since by estimating the matrix elements from atomic values or treating their ratio as a free parameter it provides a prescription for comparing theoretical results for the core-hole screening LDOS with experimental Auger profiles. It is particularly useful for analysing KLV transitions where the fact that the $\mathrm{KL}_{2,3} \mathrm{~V}$ profile approximates to $D_{\mathrm{p}}^{1}(E)$ and the $\mathrm{KL}_{1} \mathrm{~V}$ profile approximates to $D_{\mathrm{s}}^{1}(E)+D_{\mathrm{p}}^{1}(E)$ means that a crude superposition of the two profiles yields an appreciation of the extent to which the screening of the final state core-hole distorts $D_{\mathrm{s}}^{1}(E)$ $[113,114]$.

In applying (19) to the analysis of CCV Auger profiles it is usual to make use of the values of Auger matrix elements deduced from atomic structure calculations. As is made clear by (15) there are two contributions to such matrix elements; the radial integrals, which are usually evaluated from wavefunctions obtained within the frozen orbital approximation from an atomic structure calculation for the core-ionised initial state, and an evaluation of the Clebsch-Gordon algebra and angular momentum coupling contributions of the electron states involved in the transition. As discussed earlier for CVV transitions a reasonable argument can be made for employing atomic radial integrals in an analysis of CCV Auger profiles observed from solids. The angular momentum considerations are more difficult to deal with since, with the exception of the noble gases, atomic calculations for such processes necessarily involve incompletely filled valence shells, resulting in very complex expressions [58, 59]. Furthermore the local valence configuration is expected to change in the solid and indeed one of the reasons for studying Auger processes involving valence levels is to obtain information on these changes in configuration. This dependence of the matrix elements on the local valence configuration has been exploited for $\mathrm{Mg}$ [115] by varying the local valence screening configuration between $3 s^{2} 3 p^{0}, 3 s^{1} 3 p^{1} 3 s^{2} 3 p^{1}, 3 s^{1} 3 p^{2}$ and $3 s^{2} 3 p^{2}$ and calculating the relative intensities of valence $s$ and $p$ contributions to the intensities of the KLV transitions. It is found that the results for the relative intensities depend much more strongly on the assumed form of the valence configuration than on the atomic number, supporting the idea that Auger intensity ratios will be more influenced by the change in valence configuration in the solid than by changes in the radial wavefunctions of the valence electrons. A comparison of the results of these calculations with the observed contributions of the $D_{\mathrm{s}}^{1}(E)$ and $D_{\mathrm{p}}^{1}(E)$ LDOS to the KLV profiles of metallic $\mathrm{Mg}$ suggests that core-holes in metallic $\mathrm{Mg}$ are screened by electrons of $3 \mathrm{p}$ character, the assumption of $3 \mathrm{~s}$ screening yields results which are incompatible with experiment [115]. We thus have a model for metallic $\mathrm{Mg}$ in which the atomic $3 \mathrm{~s}^{2}$ configuration becomes $3 \mathrm{~s}^{1} 3 \mathrm{p}^{1}$ in 
the ground state and that the screening response to a core-ionised site generates a local $3 \mathrm{~s}^{1} 3 \mathrm{p}^{2}$ configuration. The conclusion of $\mathrm{p}$ screening in metallic $\mathrm{Mg}$ is in agreement with the results of calculations by Almbladh and co-workers $[80,81]$ but disagrees with the results of studies of the CVV profiles of metallic $\mathrm{Mg}$ [76]. Almbladh et al. [80,81] argue that this disagreement arises from an underestimate of the amount of screening charge in p orbitals in the CVV study [76]. This approach has been extended [116] to the study of the complicated spectral profiles observed for the $\mathrm{KL}_{2,3}-\mathrm{L}_{2,3} \mathrm{~L}_{2,3} \mathrm{~V}$ Auger satellites of metallic $\mathrm{Mg}$ and $\mathrm{Al}$ where it gives a satisfactory explanation of the $\mathrm{Al}$ spectrum but is unable to account for one of the features in the $\mathrm{Mg}$ spectrum.

The distortions in the LDOS of $s$ and $p$ character induced by a core-hole can be modelled in terms of an on-site Coulomb interaction, $U_{\mathrm{l}}$, between the core-hole and the conduction band DOS of 1 character. Cini [117] has shown that for filled bands the relationship between the ground state LDOS, $D_{1}^{0}(E)$, and the LDOS at the core-ionised site, $D_{1}^{1}(E)$, can be represented by;

$$
D_{1}^{1}(E)=D_{1}^{0}(E) /\left\{\left[1-U_{1} H(E)\right]^{2}+\pi^{2} U_{1}^{2} D_{1}^{0}(E)^{2}\right\}
$$

where $H(E)$ is the Hilbert transform of $D_{1}^{0}(E)$. It has been shown $[110,111]$ that this expression is able to reproduce the results of first principles calculations of the LDOS in the presence of core-holes for reasonable values of the on-site Coulomb interaction for the simple metals $\mathrm{Na}, \mathrm{Mg}$ and $\mathrm{Al}$.

The $\mathrm{KL}_{1} \mathrm{~V}$ and $\mathrm{KL}_{2,3} \mathrm{~V}$ spectra of $\mathrm{Mg}$ and its alloys with $\mathrm{Al}$ and $\mathrm{Li}$ have shown that the extent to which $D_{\mathrm{s}}^{1}(E)$ is distorted to screen a core-hole is sensitive to the electronic structure of the alloy. As $\mathrm{Al}$ is added to $\mathrm{Mg}$ the peak in $D_{\mathrm{s}}^{1}(E)$ loses intensity and eventually disappears [112, 118]. In LiMg however the screening peak is strongly enhanced [114]. This is shown by the experimental profiles of the $\mathrm{KL}_{1} \mathrm{~V}$ transitions of $\mathrm{Mg}$ in $\mathrm{Al}_{85} \mathrm{Mg}_{15}, \mathrm{Mg}$ metal and $\mathrm{Li}_{85} \mathrm{Mg}_{15}$ which are represented by the dots in Figure 5. The double peaked structure of the $\mathrm{KL}_{1} \mathrm{~V}$ profile can be clearly seen for the spectrum observed from metallic $\mathrm{Mg}$, the most bound peak arising from $D_{\mathrm{s}}^{1}(E)$. This peak is much more prominent in the spectrum observed from $\mathrm{Li}_{85} \mathrm{Mg}_{15}$ than in $\mathrm{Mg}$ and is absent from the spectrum observed from $\mathrm{Al}_{85} \mathrm{Mg}_{15}$. A simple understanding of these spectral profiles can be obtained $[113,114]$ by a consideration of the equivalent cores approximation which leads us to expect that the LDOS around a core-ionised $\mathrm{Mg}$ site in pure $\mathrm{Mg}$ will be the same as that of an $\mathrm{Al}$ impurity in $\mathrm{Mg}$. The need to screen an extra core-charge causes a peak to develop towards the bottom of the conduction band in $D_{\mathrm{s}}^{1}(E)$ resulting in a double peaked $\mathrm{KL}_{1} \mathrm{~V}$ profile in $\mathrm{Mg}$. In the $\mathrm{Al}$ rich alloy there will be little difference between the local potential of the core-ionised $\mathrm{Mg}$ site and a host $\mathrm{Al}$ site and the screening peak will not appear in $D_{\mathrm{s}}^{1}(E)$. However in the $\mathrm{Li}$ rich alloy a core-ionised $\mathrm{Mg}$ site will differ by two core charges from that of a Li host site and the screening peak will become pronounced as is observed.

As a final comment on the insight into the nature of local screening configurations that can be deduced from the use of approximate methods of analysing CCV Auger spectra we should note that a judicious combination of Auger and X-ray emission profiles observed from simple metals and alloys can be combined with the equivalent cores approximation to yield an empirical method of studying the dependence of the LDOS on the local charge on a site [113]. Such a study shows that while the shape of the LDOS of p character is not distorted in Mg by the presence of a single core-hole the presence of two core-holes does distort the $\mathrm{p}$ LDOS and results in the maximum intensity of $D_{\mathrm{p}}^{2}(E)$ being pulled to higher binding energy of the Fermi energy [113]. Studies of the $\mathrm{KL}_{2,3}-\mathrm{L}_{2,3} \mathrm{~L}_{2,3} \mathrm{~V}$ Auger satellites of metallic $\mathrm{Mg}$ and $\mathrm{Al}$ [116] confirm the general expectation that double core-hole states create more severe distortion of the LDOS than single core-hole states.

A sophisticated analysis of the spectra shown in Figure 5 requires a theoretical treatment similar to that originally developed for $\mathrm{Na}$ by von Barth and Grossmann [105]. This has recently been 
obtained by self-consistent field calculations of the local electronic structure within an embedding scheme $[112,119]$ together with an evaluation of the Auger matrix elements. The results obtained [112] at the various stages of such a calculation for pure $\mathrm{Mg}$ are shown in Figure 6 where the top panel, (a), gives the total LDOS and its decomposition into states of $\mathrm{s}$ and $\mathrm{p}$ character in the ground state. The introduction of a local core-hole gives rise to a screening peak in the LDOS of $s$ character and an increase in the LDOS of $p$ character (b). Finally the calculation of the appropriate matrix elements generates the basic theoretical profile for the $\mathrm{KL}_{1} \mathrm{~V}$ transition shown in (c). It is important to note that this calculation does not follow the form of (19) in that the matrix elements have not been separated from the LDOS and their explicit dependence on both the Auger kinetic energy and the energy of the conduction band electrons is included in the calculation. The significance of this absence of the usual simplifications can be seen in the difference between the results for the LDOS shown in (b) and the spectra including the matrix elements shown in (c). This comparison shows that whereas the overall spectral profile is relatively insensitive to a sophisticated calculation of matrix elements these do have a strong influence on the relative importance of the $s$ and $p$ LDOS contributions as a function of binding energy. In order to compare the theoretical profile of Figure $6 \mathrm{c}$ with the experimental results it is necessary to include in the theoretical profile the lifetime broadening contributions of the initial and final core-hole states, the experimental broadening and a contribution from the background of scattered electrons, all of which are reasonably well understood. The final theoretical profile is shown by the full line in Figure $5 \mathrm{~b}$ and is in good agreement with experiment. The embedding technique has also been applied [119] to the calculation of the $\mathrm{KL}_{1} \mathrm{~V}$ spectral profiles of the alloys $\mathrm{Al}_{85} \mathrm{Mg}_{15}$ and $\mathrm{Li}_{85} \mathrm{Mg}_{15}$ and the results for these systems, shown by the full lines in Figure 5a and c, are also in good agreement with experiment. The important point about the success of these calculations is not that they reproduce the Auger profiles, though this is satisfying, but that the agreement with experiment confirms that the calculation is successfully reproducing the main features of the local electronic structure in the vicinity of core-ionised sites in all three metals.

It should be noted that the embedding scheme is less successful in calculating the $\mathrm{KL}_{2,3} \mathrm{~V}$ spectral profile of these three metals $[112,119]$. The $\mathrm{Mg} \mathrm{KL}_{2,3} \mathrm{~V}$ profiles show a similar trend with $\mathrm{Mg}$ composition to that observed for the $\mathrm{KL}_{1} \mathrm{~V}$ profiles. However although this trend is reproduced by the theory the latter significantly overestimates the sharpness of the $D_{\mathrm{s}}^{1}(E)$ screening peak in all three profiles. This failure may be due to a significant lifetime broadening of the hole state in the conduction band, the effect of the neglect of which is disguised in the $\mathrm{KL}_{1} \mathrm{~V}$ profile by the large lifetime broadening of the $\mathrm{L}_{1}$ state which can decay by Coster-Kronig transitions. Alternatively the failure may be a result of the neglect in the embedding scheme of multiple scattering by the crystal lattice which can change the balance of valence $s$ and $p$ contributions to the LDOS.

The fact that the theoretical results are able to explain the experimental profiles of the $\mathrm{Mg}$ $\mathrm{KL}_{1} \mathrm{~V}$ transitions shown in Figure 6 suggests that such spectra are little influenced by the crystal potential since the embedding scheme is essentially a calculation for a spherical electron gas which does not take account of translational symmetry. The expectation that the translational symmetry of the crystal potential has only a weak effect on the KLV profiles of simple metals is confirmed by high resolution measurements of the KLV spectra of $\mathrm{Al}$ obtained by Waddington $[15,120]$ which reveal weak shoulders at the top of the peak rising from the Fermi energy similar in magnitude to the effect of the crystal potential on the valence band DOS measured in XPS [106, 107].

The success of the embedding scheme in reproducing the $\mathrm{KL}_{1} \mathrm{~V}$ profiles of $\mathrm{Mg}$ in the alloys with $\mathrm{Li}$ and $\mathrm{Al}$ (Fig. 6) makes it possible to evaluate the accuracy of the approximations usually made in analysing such profiles by means of $(19)[112,119]$. We have already noted the errors in assessing the energy dependence of the $D_{\mathrm{s}}^{1}(E)$ and $D_{\mathrm{p}}^{1}(E)$ contributions to the KLV profiles that arise from the neglect of the energy dependence of the matrix elements (Fig. 5b and c). We can also evaluate the implicit assumption that the matrix elements, and more particularly their ratio, 


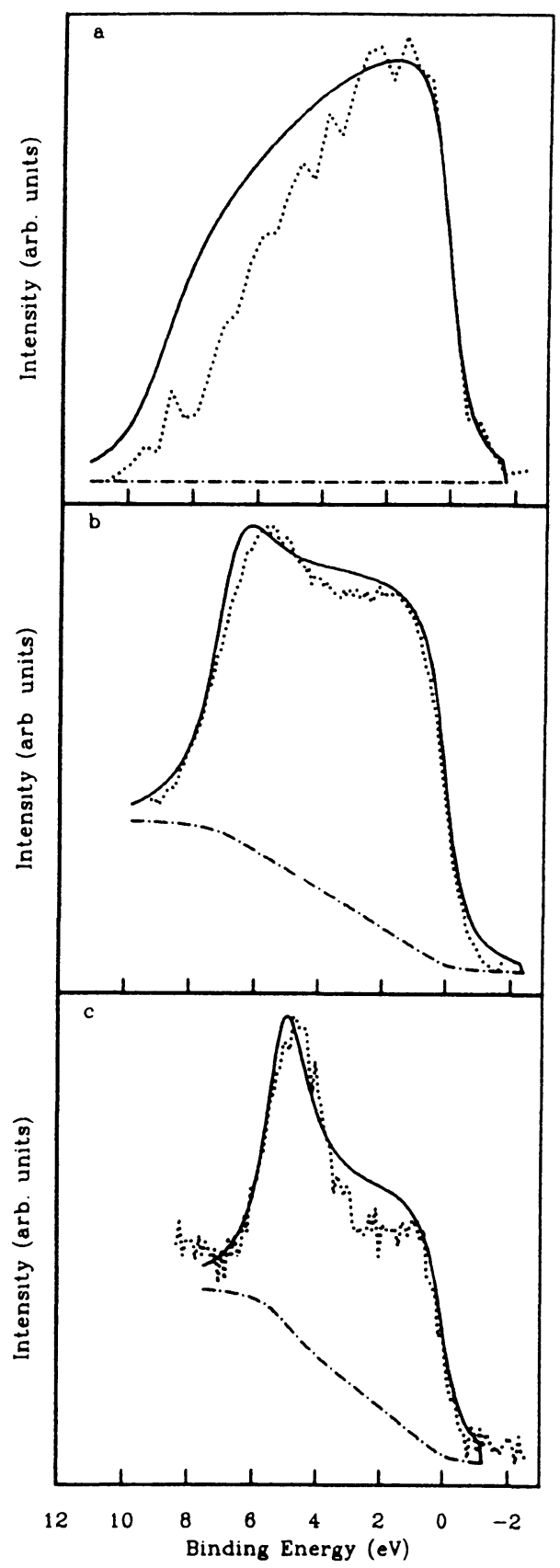

Fig. 5. - The dots in panels a, b and $c$ show the experimental profile of the $\mathrm{Mg} \mathrm{KL}_{1} \mathrm{~V}$ Auger transitions observed from $\mathrm{Al}_{85} \mathrm{Mg}_{15}$ [118], metallic $\mathrm{Mg}$ and $\mathrm{Li}_{85} \mathrm{Mg}_{15}$ [114] respectively. The lines show the results of the theoretical calculations of Fowles et al. [119].

are independent of the atomic environment. A detailed analysis shows that this simple assumption does not hold for $\mathrm{Mg}$. It is found [119] that the ratio of the transitions rates is as strongly dependent 


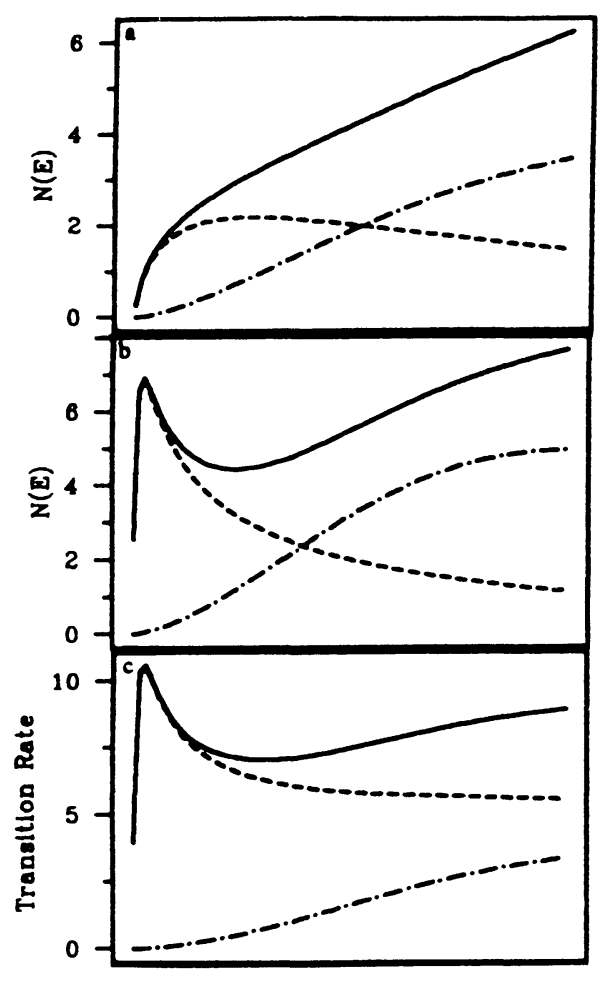

Fig. 6. - The calculated profiles of metallic $\mathrm{Mg}$ for a) the local density of states (LDOS) of the ground state, b) the LDOS of a core-ionised site and c) the $\mathrm{KL}_{1} \mathrm{~V}$ transition. In each figure the full curve shows either the total LDOS or the total Auger profile while the contributions from valence $s$ and $p$ states are shown by the dashed and dot-dashed curves respectively. The figure is taken from Fowles et al. [112].

on the local environment as the LDOS of $\mathrm{s}$ and $\mathrm{p}$ character. Fortunately it is possible to make some simplifications of the full first principles calculation and an approximate treatment [112] which allows for the variation of the matrix elements with the energy of the valence electron but neglects their dependence on the kinetic energy of the Auger electron gives quite good agreement with the Mg Auger profile and its dependence on the LDOS of $s$ and $p$ character.

The study of the LiMg and AlMg systems gives us confidence that first principles calculations are capable of explaining the KLV Auger profiles of simple metals and hence give a good representation of the local screening of core-ionised sites and its dependence on electronic structure. The approximate treatments, when combined with the equivalent cores approximation, which allows us to relate core-ionised sites to impurity sites of the next element, also provide insight into the main features of the screening LDOS, notably the sensitivity of the screening peak in $D_{\mathrm{s}}^{1}(E)$ to the difference in core-charge between the site being screened and an average site. We expect that the more complex changes in electronic structure that are produced when simple metals are alloyed with $\mathrm{d}$ band elements will also influence KLV Auger transitions and that a study of their spectral profiles will provide insight into the electronic structure of such systems. This is indeed the case as is shown by experimental results for $\mathrm{Mg}$ alloyed with a large number of $\mathrm{d}$ band elements $[118,121]$. The KLV lineshapes of Mg change radically in such alloys with new features introduced into the experimental Auger profiles which can be attributed to the hybridization of the LDOS of $s$ and $p$ character with the $d$ band $[118,121,122]$. Figure 7 shows an example of 
the effects on the Mg KLV profiles that result from alloying with $\mathrm{Ag}$ and Pd [118, 121]. The XPS study of the conduction bands of $\mathrm{MgPd}$ and $\mathrm{MgAg}$ show that the $\mathrm{Pd}$ and $\mathrm{Ag} \mathrm{d}$ bands appear at different binding energies, $\sim 4 \mathrm{eV}$ and $6 \mathrm{eV}$ respectively. The $\mathrm{d}$ band will introduce resonances in the $\mathrm{Mg} s$ and $\mathrm{p} \mathrm{LDOS}$ and these give rise to dips in the profiles of the $\mathrm{Mg} \mathrm{KL}_{2,3} \mathrm{~V}$ profiles at the appropriate binding energies. The $\mathrm{KL}_{2,3} \mathrm{~V}$ spectra of these alloys are easier to understand than the $\mathrm{KL}_{1} \mathrm{~V}$ spectra since matrix element considerations leads us to expect that the $\mathrm{KL}_{2,3} \mathrm{~V}$ profiles will be dominated by $D_{\mathrm{p}}^{1}(E)$, the shape of which, in simple metal alloys, is less distorted by the screening of the core-hole than $D_{\mathrm{s}}^{1}(E)$. The profiles of the $\mathrm{Mg} \mathrm{KL}_{1} \mathrm{~V}$ spectra are more difficult to interpret since we expect them to show significant contributions from both $D_{\mathrm{s}}^{1}(E)$ and $D_{\mathrm{p}}^{1}(E)$ and the former may show the same sensitivity to the core-hole as observed in simple metals.

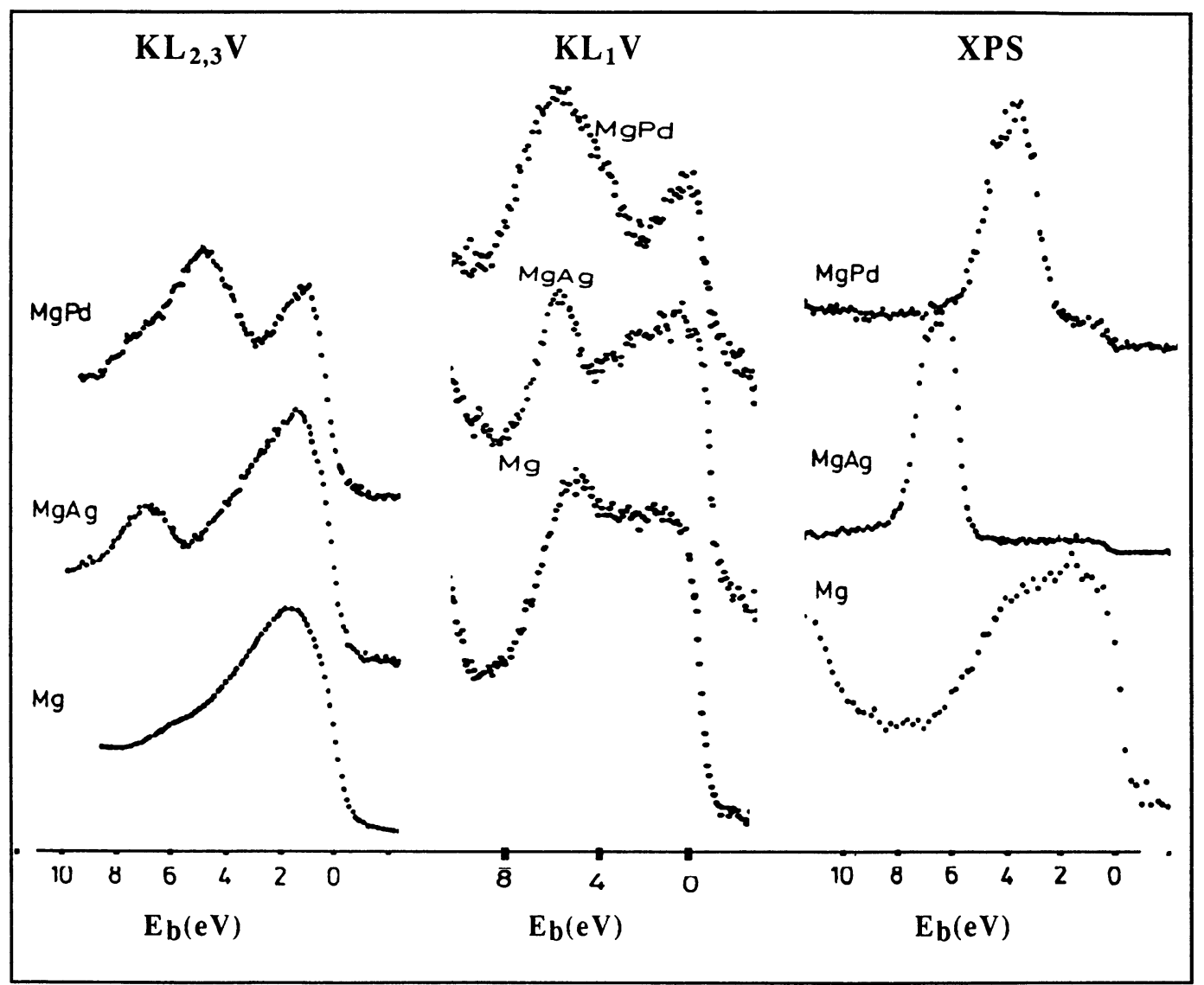

Fig. 7. - The first and second panels show the $\mathrm{Mg} \mathrm{KL}_{2,3} \mathrm{~V}$ and $\mathrm{Mg} \mathrm{KL}$ Auger spectra of the conduction band of $\mathrm{Mg}, \mathrm{AgMg}$ and PdMg respectively. The third panel shows the XPS spectra of the conduction bands observed from these metals. The results are taken from Davies and Weightman [118] and Davies et al. [121].

Recently Kucherenko and Perlov [122] have carried out a theoretical study of the Mg KLV spectra of MgPd alloys within a linear muffin tin orbital-Green function approach. Overall they find that the screening of a core-ionised $\mathrm{Mg}$ site is more delocalised in these alloys than in simple metal systems with more than $10 \%$ of the screening charge being localized outside the Mg atomic 
sphere at the neighboring Pd atoms. They find that the LDOS of $\mathrm{s}$ character does not hybridise strongly with the.Pd d band in the ground state in these systems and that $D_{\mathrm{s}}^{1}(E)$ shows a similar response to a $\mathrm{Mg}$ core-hole to that observed in metallic $\mathrm{Mg}$. However the $\mathrm{Mg} \mathrm{LDOS}$ of $\mathrm{p}$ character does hybridise strongly with the Pd $\mathrm{d}$ band and this results in a different screening response of $\mathrm{p}$ electrons to that observed in the simple metals. Whereas in simple metals core-hole screening leads to an even increase in $D_{\mathrm{p}}^{\mathbf{1}}(E)$ across the conduction band, in MgPd alloys the increase in $D_{\mathrm{p}}^{1}(E)$, which accounts for most of the additional screening, is concentrated to high binding energy of the $\mathrm{d}$ band resonance towards the bottom of the band. This latter result contrasts with the results of an equivalent cores analysis of the screening contribution of $D_{\mathrm{p}}^{1}(E)$ to core-ionised sites in $\mathrm{MgCu}$ alloys [75] which suggested that in these alloys the screening response is concentrated to lower binding energy of the $d$ band resonance and towards the Fermi energy. It is possible that the binding energy of the $d$ band resonance has an important influence on the screening response of $\mathrm{Mg}$ electrons of $\mathrm{s}$ and $\mathrm{p}$ character since its position will influence the extent to which each hybridises with the $\mathrm{d}$ band in the ground state. Further, as shown by the results for MgPd [122], the $d$ band itself may make an important screening contribution. This latter possibility might account for a puzzling anomaly in the Mg KLV spectra of MgZn alloys [15, 118] where it is observed that as the $\mathrm{Zn}$ concentration increase the peak in the LDOS of s character, which is prominent in the $\mathrm{KL}_{1} \mathrm{~V}$ spectrum of metallic $\mathrm{Mg}$, is reduced in intensity and is absent from the spectrum of $\mathrm{MgZn}$. This results in very similar profiles for the $\mathrm{Mg} \mathrm{KL_{1 }} \mathrm{V}$ and $\mathrm{KL}_{2,3} \mathrm{~V}$ transitions of $\mathrm{MgZn}[118,121]$ indicating that the screening of the core-hole does not distort either the $D_{\mathbf{s}}^{1}(E)$ or the $D_{\mathrm{p}}^{1}(E)$ LDOS. In these alloys the $\mathrm{Zn} \mathrm{d}$ band is probably too bound to hybridise with the $\mathrm{Mg}$ LDOS but it may make an important contribution to the screening of a $\mathrm{Mg}$ core-hole state.

\section{Conclusions}

The development of Auger spectroscopy as a probe of local electronic structure has been described. This aspect of Auger spectroscopy developed more slowly than the use of Auger spectroscopy for elemental analysis since although both techniques resulted from advances in the design of electron spectrometers the former was particularly dependent on the development of high performance instruments capable of making high resolution measurements of weak transitions. Another limitation on progress in this aspect of Auger spectroscopy was its dependence on the theoretical work necessary to interpret Auger profiles.

This review has shown that these limitations have now been overcome and that Auger spectroscopy is in the process of reaching its full potential as a probe of local electronic structure. In recent years high performance electron spectrometers capable of yielding high resolution measurements of the profiles of Auger transitions involving valence levels have been constructed and both first principles and approximate theoretical techniques have reached the stage at which they can be used routinely to extract the important information on local electronic structure that is contained in Auger profiles. The review has shown that the study of CCC, CCV and CVV Auger transitions can reveal useful information on local electronic structure provided the experimental results are interpreted in the appropriate theoretical context. The final state rule is particularly useful in establishing a framework for the interpretation of CVV and CCV profiles in the absence of electron correlation in the final state and it has been shown that the study of the spectral profiles of such transitions can reveal the nature of the screening configuration and the LDOS and its dependence on electronic structure for core-ionised sites. Studies of the Mg KLV transitions of alloys between $\mathrm{Mg}$ and $\mathrm{d}$ band elements have shown that the spectral profiles contain information on the hybridisation between the $d$ band and the $s$ and $p$ bands and on the nature of electron screening. 
The Auger spectra of transitions that create final states in otherwise filled $\mathrm{d}$ bands can provide unique insight into the magnitude of on-site electron correlation and there is evidence that such transitions are also sensitive to off-site interactions between the final state $d$ holes. Unfortunately we do not yet have a complete theory of the Auger spectra of transitions involving incompletely filled d bands.

It has been shown that the analysis of environmentally determined Auger parameter shifts, formed by combining shifts in the kinetic energies of CCC Auger transitions and in the binding energies of core levels measured by XPS, can be used to separate and evaluate contributions from initial and final state effects. This has lead to the development of a potential model relating the potential in an atomic core to the valence charge and to a new scale of electronegativity for elements of the first two rows of the periodic table. While Auger parameter studies give insight into the extent of charge transfer and the nature of core-hole screening in metal alloys there is a need for further theoretical work if they are to reach their full potential for the study of semiconductors and insulators where the screening is more delocalised.

\section{References}

[1] Auger P., J. Phys. Radium 6 (1925) 205.

[2] Burhop E.H.S., "The Auger effect and other radiationless transitions" (Cambridge University Press, 1952).

[3] Sieghbahn K., Nordling C., Fahlman A., Nordberg R., Hamrin K., Hedman J., Johansson G., Bergmark T., Karlsson S.E., Lindgren I. and Lindberg B., Nova Acta Regiae Soc. Sci. Upsaliensis, Ser. IV 20 (1967).

[4] ESCA Applied to Free Molecules, Siegbahn K., Nordling C., Johansson G., Hedman J., Hedin P.F., Hamrin K., Gelius U., Bergmark T., Werme L.O., Mann R. and Baer Y. (North-Holland, Amsterdam, 1969).

[5] Carlson T.A., "Photoelectron and Auger Spectroscopy" (Plenum, New York, 1975).

[6] Weightman P., Rep. Prog. Phys. 45 (1982) 753.

[7] Evans J.A., Laine A.D., Fowles P.S., Duo L., McGilp J.F., Mondio G., Norman D. and Weightman P., J. Phys. Condens. Matter 2 (1990) 195.

[8] Gelius U., Basilier E., Svensson S., Bergmark T. and Siegbahn K., J. Electron Spectrosc. 2 (1973) 405.

[9] Weightman P. and Andrews P.T., J. Phys. C 13 (1980) 3529.

[10] Weightman P., Phys. Scr. T41 (1992) 277.

[11] Thomsen M., Baker M.D., Christie A. and Tyson J.F., "Auger Electron Spectroscopy" (Wiley, New York, 1985).

[12] C.L. Briant and R.P. Messmer Eds. "Auger electron spectroscopy" (Academic Press, London, 1988).

[13] G. Cubiotti, G. Mondio and K. Wandelt Eds. "Auger Spectroscopy and Electronic Structure" (Springer-Verlag, Berlin, 1989).

[14] Weightman P., Phys. Scr. T25 (1989) 165.

[15] Weightman P., J. Electron Spectrosc. 68 (1994) 127.

[16] Gunarson O. and Schonhammer K., Phys. Rev. B 22 (1980) 3710.

[17] Drchal V. and Cini M., J. Phys. Cond. Matter 6 (1994) 8549.

[18] Mehlhorn W., Z. Phys. 208 (1968) 1.

[19] Larkins F.P., J. Phys. B 4 (1971) 1.

[20] Larkins F.P., J. Phys. B 4 (1971) 14.

[21] Kelly H.P., Phys. Rev. A 11 (1975) 556.

[22] Larkins F.P., in "Atomic Inner Shell Processes", B. Crasemann Ed. (New York, Academic, 1975).

[23] Weightman P., J. Phys. C 9 (1976) 1117.

[24] Briancon C. and Desclaux J.P., Phys. Rev. A 13 (1976) 2157.

[25] Chen M.H., Crasemann B., Aoyagi and Mark H., Phys. Rev. A 20 (1979) 385.

[26] Krause M.O. and Nestor C.W., Phys. Scr. 16 (1977) 285.

[27] Thomas T.D., J. Electron Spectrosc., Rel. Phen. 20 (1980) 117. 
[28] Wagner C.D., Farad. Discuss. Chem. Soc. 60 (1975) 291.

[29] Waddington S.D., in "Practical Surface Analysis" Vol. 1, 2nd edition; D. Briggs and M.P. Seah Eds. (Wiley, 1990).

[30] Evans J.A., Laine A.D., Weightman P., Matthew J.A.D., Woolf D.A., Westwood D.I. and Williams R.H., Phys. Rev. B 46 (1992) 1513.

[31] Cole R.J. and Weightman P., Surf. Rev. Lett. 1 (1994) 469.

[32] Cole R.J., Weightman P. and Matthew J.A.D., J. Electron Spectrosc. 72 (1995) 255.

[33] Thomas T.D. and Weightman P., Phys. Rev. B 33 (1986) 5406.

[34] Gelius U., Phys. Scr. 9 (1973) 133.

[35] Cole R.J. and Weightman P., in "Metallic Alloys: Experimental and Theoretical Perspectives", J.S. Faulkner and R.G. Jordan Eds. (Kluwer, The Netherlands, 1994) 27.

[36] Walker C.G.H., Morton S.A., Beamson G., Matthew J.A.D. and Yousif F.N., J. Electron Spectrosc. 70 (1994) 73.

[37] Waddington S.D., Weightman P., Matthew J.A.D. and Gcrassie A.D.C., Phys. Rev. B 39 (1989) 10239.

[38] Matthew J.A.D., Weightman P. and Waddington S.D., J. Phys. Condens. Matter 1 (1989) SB217.

[39] Cole R.J., Evans J.A., Weightman P., Matthew J.A.D., Woolf D.A. and Westwood D.I., Phys. Rev. B 49 (1994) 7528.

[40] Wagner C.D. and Bileon P., Surf. Sci. 35 (1973) 82.

[41] Moretti G. and Porta P., Surf. Interface Anal. 15 (1990) 47.

[42] Moretti G., Surf. Interface Anal. 16 (1990) 159.

[43] Hodges L., Watson R.E. and Ehrenreich H., Phys. Rev. B 5 (1972) 3953.

[44] Cole R.J., Gregory D.A.C. and Weightman P., Phys. Rev. B 49 (1994) 5657.

[45] Snyder L.C., J. Chem. Phys. 55 (1971) 95.

[46] Saethre L.J., Siggel M.R.F. and Thomas T.D., J. Am. Chem. Soc. 113 (1991) 5224.

[47] Cole R.J. and Weightman P., J. Phys. Condens. Matter 6 (1994) 5783.

[48] Jackson M.D., Cole R.J., Brookes N.J. and Weightman P., J. Electron Spectrosc. 72 (1995) 261.

[49] Pauling L., "The Nature of the Chemical Bond" (Cornell, New York, 1960).

[50] Cole R.J., Brooks N.J., Weightman P. and Matthew J.A.D., Phys. Rev. B, in press.

[51] Ley L., Kowalczyk S.P., McFeeley R., Pollack R.A. and Shirley D.A., Phys. Rev. B 8 (1973) 2302.

[52] Williams A.R. and Lang N.D., Phys. Rev. Lett. 40 (1978) 954.

[53] Slater J.C., "Quantum Theory of Atomic Structure” (McGraw-Hill, New York, 1960).

[54] Asaad W.N. and Burhop E.H.S., Proc. Phys. Soc. 71 (1958) 369.

[55] Assad W.N. and Mehlhorn W., Z. Phys. 217 (1968) 304.

[56] Mehlhorn W. and Asaad W.N., Z. Phys. 191 (1966) 231.

[57] Aoyagi M., Chen M.H., Crasemann B., Huang K.N. and Mark H., NASA Technical Memorandum, NASA TM X-73 (1976) 148.

[58] McGuire E.J., in “Atomic Inner Shell Processes”, B. Crasemann Ed. (Academic, New York, 1975).

[59] Cubiotti G., Laine A. and Weightman P., J. Phys. Condens. Matter 1 (1989) 7, 723.

[60] El Ibyari S.N., Asaad W.N. and McGuire E.J, Phys. Rev. A 5 (1976) 150.

[61] Herman F. and Skillman S., "Atomic Structure Calculations" (Prentice-Hall, Englewood Cliffs, NJ, 1963).

[62] Chen M.H. and Crasemann B., Phys. Rev. A 8 (1973) 7.

[63] Crasemann B., in "Atomic Inner Shell Processes", B. Crasemann Ed. (New York, Academic, 1975).

[64] Chen M.H., Crasemann B. and Mark H., At. Data Nucl. Data Tables 24 (1979) 13.

[65] Chen M.H., Crasemann B. and Mark H., Phys. Rev. A 21 (1980) 442.

[66] Chen M.H., Crasemann B. and Mark H., Phys. Rev. A 27 (1983) 2989.

[67] Vayrynen J., Aksela S., Kellokumpu M. and Aksela H., Phys. Rev. B 22 (1980) 1610.

[68] Aksela S., Aksela H., Vuontisjarvi M., Vayrynen J. and Lahteenkorva E., J. Electron Spectroscop. 11 (1977) 137.

[69] Parry-Jones A.C., Weightman P. and Andrews P., J. Phys. C 12 (1979) 1587.

[70] Jennison D.R., Phys. Rev. Lett. 40 (1978) 807.

[71] von Barth U. and Grossman G., Phys. Scri. 28 (1983) 107.

[72] von Barth U. and Grossman G., Phys. Rev. B 25 (1982) 5150.

[73] Ramaker D.E., Phys. Rev. B 25 (1982) 7341.

[74] Jennison D.R., Madden H.H. and Zehner D.M., Phys. Rev. B 21 (1980) 430.

[75] Weightman P., in "Auger Spectroscopy and Electronic Structure", G. Cubiotti, G. Mondio and K. Wandelt Eds. (Springer-Verlag, Berlin, 1989).

[76] Davies M., Weightman P. and Jennison D.R., Phys. Rev. B 29 (1984) 5313. 
[77] Tougaard S., Phys. Rev. B 34 (1986) 6779.

[78] Ramaker D.E., Hutson F.L., Turner N.H. and Mei W.N., Phys. Rev. B 33 (1986) 2574.

[79] Jennison D.R., Madden H.H. and Zehner D.M., Phys. Rev. B 21 (1980) 430.

[80] Almbladh C.O., Morales A.L. and Grossmann G., Phys. Rev. B 39 (1989) 3489.

[81] Almbladh C.O. and Morales A.L., Phys. Rev. B 39 (1989) 3503.

[82] Cini M., Pernaselci A. and Paparazzo E., J. Electron. Spectrosc. 72 (1995) 77.

[83] Powell C.J., Phys. Rev. Lett. 30 (1973) 1179.

[84] Cini M., Solid State Commun. 20 (1976) 605.

[85] Cini M., Solid State Commun. 24 (1977) 681.

[86] Sawatzky G.A., Phys. Rev. Lett. 39 (1977) 504.

[87] Cini M. and D'Andrea A., J. Phys. C 16 (1983) 4469.

[88] Weightman P., Andrews P.T., Stocks G.M. and Winter H., J. Phys. C 16 (1983) L81.

[89] Weightman P., Wright H., Waddington S.D., v. d. Marel D., Sawatzky G.A., Diakun G.P. and Norman D., Fhys. Rev. B 36 (1987) 9098.

[90] Hannah P.H. and Weightman P., J. Phys. F 16 (1986) 1015.

[91] Vos M., Sawatzky G.A., Davies M., Weightman P. and Andrews P.T., Solid State Commun. 52 (1984) 159.

[92] Nahm T.U., Han M., Oh S.J., Park J.H., Allen J.W. and Chung S.M., Phys. Rev. Lett. 70 (1993) 3663.

[93] Weightman P., Cole R.J., Verdozzi C. and Durham P.J., Phys. Rev. Lett. 72 (1994) 793.

[94] Unsworth P., Brooks N., Thornton J.M.C., Sancrotti M., D’Addato S., Duo L., Andrews P.T. and Weightman P., J. Electron Spectrosc. 72 (1995) 217.

[95] Jennison D.R., Hillebrecht F.U. and Fuggle J.C., J. Vac. Sci. Technol. A2 (1983) 1049.

[96] Drchal V. and Cini M., J. Phys. Condens. Matter 6 (1994) 8549.

[97] Kotrla M. and Drchal V., J. Phys. Condens. Matter 17 (1992) 4251.

[98] Cini M. and Verdozzi C., J. Phys. Condens. Matter. 1 (1989) 7457.

[99] Aksela S., Pekkala T., Aksela H., Wallenius M. and Harkoma M., Phys. Rev. B 35 (1987) 1, 426.

[100] Yin L.I., Tsang T. and Adler I., Phys. Rev. B 15 (1977) 2974.

[101] Verdozzi C., Cini M., McGilp J.F., Mondio G., Norman D., Evans J.A., Laine A.D., Fowles P.S., Duo L. and Weightman P., Phys. Rev. B 43 (1991) 9550.

[102] Verdozzi C., Cini M., Evans J.A., Cole R.J., Laine A.D., Fowles P.S., Duo L. and Weightman P., European Phys. Lett. 16 (1991) 743.

[103] Verdozzi C. and Cini M., Phys. Rev. B 51 (1995) 7412.

[104] Cole R.J., Verdozzi C., Cini M. and Weightman P., Phys. Rev. B 49 (1994) 13329.

[105] von Barth U. and Grossman G., Phys. Scr. 28 (1983) 107.

[106] Steiner P., Hochst H. and Hufner S., in "Topics in Applied Physics", vol. 27, M. Cardona and L. Ley Eds. (Springer-Verlag, Berlin, 1979) 349.

[107] Davies M., Hannah P.H., Weightman P. and Andrews P.T., J. Phys. F 14 (1984) 355.

[108] Barrie A. and Street F.J., J. Electron Spectrosc. 7 (1975) 1.

[109] Feibelman P.J. and McGuire E.J., Phys. Rev. B 15 (1977) 3006.

[110] Kucherenko Y.N., J. Electron Spectrosc. 53 (1990) 39.

[111] Kucherenko Y.N. and Perlov A.Y., J. Electron Spectrosc. 58 (1992) 199.

[112] Fowles P.S., Inglesfield J.E. and Weightman P., J. Phys. Condens. Matter 3 (1991) 641.

[113] Hannah P.H., Weightman P. and Andrews P.T., Phys. Rev. B 31 (1985) 6238.

[114] Fowles P.S. and Weightman P., Phys. Scr. T41 (1992) 160.

[115] Laine A.D., Cubiotti G. and Weightman P., J. Phys. Condens. Matter 2 (1990) 2421.

[116] Laine A.D., Fowles P.S., Cubiotti G., Inglesfield J.E., Waddington S.D. and Weightman P., J. Phys. Condens. Matter 2 (1990) 9949.

[117] Cini M., Phys. Rev. B 17 (1978) 2486.

[118] Davies M. and Weightman P., Phys. Rev. B 30 (1984) 4183; Erratum, Phys. Rev. B 32 (1985) 8423.

[119] Fowles P.S., Inglesfield J.E. and Weightman P., J. Phys. Condens. Matter 4 (1992) 8729.

[120] Waddington S.D., PhD Thesis, University of Liverpool (1988) unpublished.

[121] Davies M., Weightman P. and Jennison D.R., Phys. Rev. B 29 (1984) 5318.

[122] Kucherenko Y.N. and Perlov A.Y., J. Electron Spectrosc. 58 (1992) 211.

[123] Weightman P., Andrews P.T. and Parry-Jones A.C., J. Phys C 12 (1979) 3635. 